\title{
Oxidative Coupling of an Anionic Gallium(I) Carbene Analogue: Synthesis and Structural Characterization of an Unprecedented $\pi$-Cyclopentadienyl Bridged Digallane Complex
}

\section{Robert J. Baker, Cameron Jones*, Marc Kloth and Jamie A. Platts}

Center for Fundamental and Applied Main Group Chemistry, School of Chemistry, Cardiff University, Cardiff, P.O. Box 912, Park Place, Cardiff, UK, CF10 3TB

\section{SUPPLEMENTARY MATERIAL}

\section{Supplementary Calculation Data for $\left[\left\{\mathrm{Ga}[\mathrm{N}(\mathrm{Me}) \mathrm{C}(\mathrm{H})]_{2}\right\}_{2}\left\{\mu-\mathrm{CpK}\left(\mathrm{NH}_{3}\right)_{4}\right\}\right]$}

All geometries were optimized in Gaussian98 [a] using the methods recommended by Boehme and Frenking[b], namely the BP86 density functional [c] with a 6-31G(d) basis set on $\mathrm{C}, \mathrm{N}$ and $\mathrm{H}[\mathrm{d}]$ and Stuttgart-Dresden ECP/basis set on Ga and K [e], augmented by a dtype polarisation function with exponent 0.207 on Ga [f]. Atomic charges, orbital populations, and bonding analysis were obtained from the NBO scheme $[\mathrm{g}]$. The total electronic energy for the complex at this level is $-985.814070736 \mathrm{au}$.

[a] Gaussian 98, Revision A.10, M. J. Frisch, G. W. Trucks, H. B. Schlegel, G. E. Scuseria, M. A. Robb, J. R. Cheeseman, V. G. Zakrzewski, J. A. Montgomery, Jr., R. E. Stratmann, J. C. Burant, S. Dapprich, J. M. Millam, A. D. Daniels, K. N. Kudin, M. C. Strain, O. Farkas, J. Tomasi, V. Barone, M. Cossi, R. Cammi, B. Mennucci, C. Pomelli, C. Adamo, S. Clifford, J. Ochterski, G. A. Petersson, P. Y. Ayala, Q. Cui, K. Morokuma, P. Salvador, J. J. Dannenberg, D. K. Malick, A. D. Rabuck, K. Raghavachari, J. B. Foresman, J. Cioslowski, J. V. Ortiz, A. 
G. Baboul, B. B. Stefanov, G. Liu, A. Liashenko, P. Piskorz, I. Komaromi, R. Gomperts, R. L. Martin, D. J. Fox, T. Keith, M. A. Al-Laham, C. Y. Peng, A. Nanayakkara, M. Challacombe, P. M. W. Gill, B. Johnson, W. Chen, M. W. Wong, J. L. Andres, C. Gonzalez, M. Head-Gordon, E. S. Replogle, and J. A. Pople, Gaussian, Inc., Pittsburgh PA, 2001.

[b] C. Boehme and G. Frenking, Chem. Eur. J. 1999, 5, 7.

[c] i) A.D. Becke, Phys. Rev. A 1988, 38, 3098. ii) J.P. Perdew, Phys. Rev. A 1986, 33, 8822. [d] i) R. Ditchfield, W.J. Hehre, J.A. Pople, J. Chem. Phys. 1971, 54, 724, ii) W.J. Hehre, R. Ditchfield, J.A. Pople, J. Chem. Phys. 1972, 56, 2257, iii) M.S. Gordon, Chem. Phys. Lett. 1980, 76, 163, iv) P.C. Hariharan, J.A. Pople, Theor. Chim. Acta 1973, 28, 213.

[e] i) M. Dolg, U. Wedig, H. Stoll, H. Preuss, J. Chem. Phys. 1987, 86, 866, ii) A. Bergner, M. Dolg, W. Kuehle, H. Stoll, H. Preuss, Mol. Phys. 1993, 80, 1431.

[f] J. Andzelm, S. Huzinaga, M. Klobukowski, E. Radzio, Y. Sakai, H. Tatekawi, Gaussian Basis Sets for Molecular Calculations, Elsevier, Amsterdam, 1984.

[g] A. E. Reed, L. A. Curtiss, F. Weinhold, Chem. Rev. 1988, 88, 899. 
Coordinate file (.xyz) for the fully optimised geometry of $\left[\left\{\mathrm{Ga}[\mathrm{N}(\mathrm{Me}) \mathrm{C}(\mathrm{H})]_{2}\right\}_{2}\{\mu-\right.$ $\left.\left.\mathrm{CpK}\left(\mathrm{NH}_{3}\right)_{4}\right\}\right]$

\begin{tabular}{|c|c|c|c|}
\hline $\mathrm{Ga}$ & 1.626798 & -1.181914 & 0.025185 \\
\hline $\mathrm{Ga}$ & 1.547005 & 1.291016 & 0.075444 \\
\hline $\mathrm{N}$ & 1.965494 & -2.529168 & 1.358036 \\
\hline $\mathrm{N}$ & 1.875708 & 2.605249 & 1.441594 \\
\hline $\mathrm{C}$ & 1.702401 & -2.558096 & 2.782335 \\
\hline C & 1.746668 & 2.550794 & 2.883880 \\
\hline $\mathrm{N}$ & 2.284741 & -2.441822 & -1.273731 \\
\hline $\mathrm{N}$ & 1.994759 & 2.657675 & -1.204608 \\
\hline C & 2.533843 & -3.666071 & -0.640420 \\
\hline C & 2.365790 & -3.713738 & 0.721715 \\
\hline $\mathrm{C}$ & 2.228229 & 3.864136 & -0.531117 \\
\hline $\mathrm{C}$ & 2.158795 & 3.837099 & 0.838698 \\
\hline C & 2.046779 & 2.635380 & -2.653173 \\
\hline $\mathrm{C}$ & 2.428225 & -2.359758 & -2.713585 \\
\hline $\mathrm{C}$ & -1.083568 & -0.172279 & $-1 \cdot 34$ \\
\hline $\mathrm{C}$ & -1.066254 & -1.269269 & -0.4351 \\
\hline $\mathrm{C}$ & -1.236771 & -0.747048 & 0.888216 \\
\hline $\mathrm{C}$ & -1.313141 & 0.665703 & 0.795789 \\
\hline $\mathrm{C}$ & -1.204890 & 1.026777 & -0.587604 \\
\hline $\mathrm{K}$ & -4.207768 & -0.121193 & -0.121540 \\
\hline $\mathrm{H}$ & 2.315045 & 4.721926 & 1.467278 \\
\hline $\mathrm{H}$ & 2.867387 & -4.525269 & -1.234792 \\
\hline $\mathrm{H}$ & 2.548653 & -4.614471 & 1.320647 \\
\hline $\mathrm{H}$ & 2.451290 & 4.769420 & -1.108983 \\
\hline $\mathrm{H}$ & 2.270774 & -1.319304 & -3.048458 \\
\hline $\mathrm{H}$ & 1.699283 & -3.002388 & -3.261594 \\
\hline $\mathrm{H}$ & 3.444831 & -2.664287 & -3.04 \\
\hline $\mathrm{H}$ & 1.506638 & -1.534964 & 3.150556 \\
\hline $\mathrm{H}$ & 0.819329 & -3.185507 & 3.052848 \\
\hline $\mathrm{H}$ & 2.569431 & -2.955602 & 3.355097 \\
\hline $\mathrm{H}$ & 1.377658 & 3.398447 & -3.114171 \\
\hline $\mathrm{H}$ & 3.070892 & 2.818747 & -3.048314 \\
\hline $\mathrm{H}$ & 1.718721 & 1.645163 & -3.016583 \\
\hline $\mathrm{H}$ & 0.901816 & 3.168926 & 3.268390 \\
\hline $\mathrm{H}$ & 2.666628 & 2.905235 & 3.399037 \\
\hline $\mathrm{H}$ & 1.569163 & 1.510003 & 3.208287 \\
\hline $\mathrm{H}$ & -1.224337 & -1.337912 & 1.810919 \\
\hline $\mathrm{H}$ & -0.987945 & -2.328344 & -0.700819 \\
\hline $\mathrm{H}$ & -0.921930 & -0.235696 & -2.428992 \\
\hline $\mathrm{H}$ & -1.228285 & 2.044901 & -0.988711 \\
\hline $\mathrm{H}$ & -1.390349 & 1.367907 & 1.631320 \\
\hline $\mathrm{N}$ & -4.043347 & -2.968604 & 0.394533 \\
\hline $\mathrm{N}$ & -4.639343 & 0.243021 & 2.760302 \\
\hline $\mathrm{N}$ & -4.550653 & 2.759239 & -0.571421 \\
\hline $\mathrm{N}$ & -4.043402 & -0.493991 & -2.973426 \\
\hline $\mathrm{H}$ & -4.646416 & -3.789921 & 0.533051 \\
\hline $\mathrm{H}$ & -3.366089 & -3.235838 & -0.333653 \\
\hline $\mathrm{H}$ & -3.465642 & -2.889309 & 1.243926 \\
\hline $\mathrm{H}$ & -4.568145 & 1.242544 & 2.991485 \\
\hline $\mathrm{H}$ & -5.407840 & -0.104614 & 3.348383 \\
\hline $\mathrm{H}$ & -3.784813 & -0.188260 & 3.137739 \\
\hline $\mathrm{H}$ & -4.248339 & 2.944034 & -1.536793 \\
\hline $\mathrm{H}$ & -5.380517 & 3.350515 & -0.431920 \\
\hline $\mathrm{H}$ & -3.817791 & 3.169118 & 0.022311 \\
\hline $\mathrm{H}$ & -3.697558 & -1.447729 & -3.140355 \\
\hline $\mathrm{H}$ & -4.636468 & -0.265641 & -3.782467 \\
\hline & -3 . & 0.105699 & -3.054782 \\
\hline
\end{tabular}

Natural Atomic Orbital and Natural Bond Order Analyses for $\left[\left\{\mathrm{Ga}[\mathrm{N}(\mathrm{Me}) \mathrm{C}(\mathrm{H})]_{2}\right\}_{2}\{\mu-\right.$ $\left.\left.\mathrm{CpK}\left(\mathrm{NH}_{3}\right)_{4}\right\}\right]$

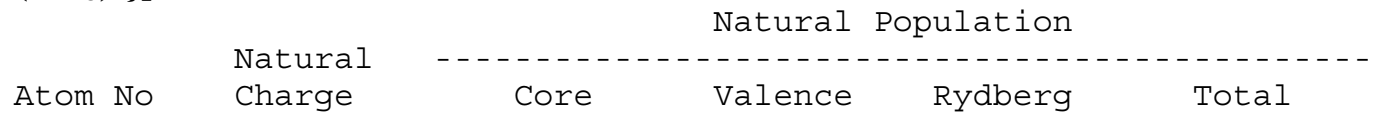




\begin{tabular}{|c|c|c|c|c|c|c|}
\hline C & 1 & -0.37027 & 1.99892 & 4.35537 & 0.01599 & 6.37027 \\
\hline C & 2 & -0.42244 & 1.99897 & 4.40616 & 0.01731 & 6.42244 \\
\hline $\mathrm{C}$ & 3 & -0.37962 & 1.99893 & 4.36496 & 0.01572 & 6.37962 \\
\hline $\mathrm{C}$ & 4 & -0.38379 & 1.99894 & 4.36886 & 0.01599 & 6.38379 \\
\hline $\mathrm{C}$ & 5 & -0.40381 & 1.99897 & 4.38833 & 0.01652 & 6.40381 \\
\hline $\mathrm{Ga}$ & 6 & 1.03015 & 28.00000 & 1.96126 & 0.00859 & 29.96985 \\
\hline $\mathrm{N}$ & 7 & -0.81524 & 1.99936 & 5.80252 & 0.01335 & 7.81524 \\
\hline $\mathrm{C}$ & 8 & -0.47822 & 1.99931 & 4.46671 & 0.01220 & 6.47822 \\
\hline $\mathrm{Ga}$ & 9 & 1.02026 & 28.00000 & 1.97155 & 0.00819 & 29.97974 \\
\hline $\mathrm{N}$ & 10 & -0.81153 & 1.99936 & 5.79890 & 0.01327 & 7.81153 \\
\hline $\mathrm{C}$ & 11 & -0.47848 & 1.99931 & 4.46696 & 0.01220 & 6.47848 \\
\hline $\mathrm{N}$ & 12 & -0.81356 & 1.99936 & 5.80094 & 0.01326 & 7.81356 \\
\hline $\mathrm{C}$ & 13 & -0.12916 & 1.99888 & 4.11225 & 0.01804 & 6.12916 \\
\hline $\mathrm{C}$ & 14 & -0.13186 & 1.99888 & 4.11495 & 0.01803 & 6.13186 \\
\hline $\mathrm{N}$ & 15 & -0.81136 & 1.99936 & 5.79869 & 0.01331 & 7.81136 \\
\hline $\mathrm{C}$ & 16 & -0.12781 & 1.99888 & 4.11091 & 0.01802 & 6.12781 \\
\hline $\mathrm{C}$ & 17 & -0.13009 & 1.99888 & 4.11312 & 0.01809 & 6.13009 \\
\hline $\mathrm{C}$ & 18 & -0.47818 & 1.99931 & 4.46666 & 0.01220 & 6.47818 \\
\hline $\mathrm{C}$ & 19 & -0.47820 & 1.99931 & 4.46662 & 0.01226 & 6.47820 \\
\hline K & 20 & 0.90078 & 17.99720 & 0.09159 & 0.01043 & 18.09922 \\
\hline $\mathrm{H}$ & 21 & 0.21501 & 0.00000 & 0.78376 & 0.00123 & 0.78499 \\
\hline $\mathrm{H}$ & 22 & 0.21539 & 0.00000 & 0.78338 & 0.00123 & 0.78461 \\
\hline $\mathrm{H}$ & 23 & 0.21499 & 0.00000 & 0.78377 & 0.00124 & 0.78501 \\
\hline $\mathrm{H}$ & 24 & 0.21516 & 0.00000 & 0.78360 & 0.00124 & 0.78484 \\
\hline $\mathrm{H}$ & 25 & 0.22598 & 0.00000 & 0.77242 & 0.00160 & 0.77402 \\
\hline $\mathrm{H}$ & 26 & 0.20067 & 0.00000 & 0.79599 & 0.00334 & 0.79933 \\
\hline $\mathrm{H}$ & 27 & 0.21510 & 0.00000 & 0.78229 & 0.00261 & 0.78490 \\
\hline $\mathrm{H}$ & 28 & 0.22562 & 0.00000 & 0.77273 & 0.00165 & 0.77438 \\
\hline $\mathrm{H}$ & 29 & 0.19652 & 0.00000 & 0.79986 & 0.00362 & 0.80348 \\
\hline $\mathrm{H}$ & 30 & 0.21597 & 0.00000 & 0.78148 & 0.00255 & 0.78403 \\
\hline $\mathrm{H}$ & 31 & 0.20338 & 0.00000 & 0.79357 & 0.00305 & 0.79662 \\
\hline $\mathrm{H}$ & 32 & 0.21410 & 0.00000 & 0.78309 & 0.00281 & 0.78590 \\
\hline $\mathrm{H}$ & 33 & 0.22462 & 0.00000 & 0.77366 & 0.00173 & 0.77538 \\
\hline $\mathrm{H}$ & 34 & 0.20012 & 0.00000 & 0.79655 & 0.00332 & 0.79988 \\
\hline $\mathrm{H}$ & 35 & 0.21535 & 0.00000 & 0.78200 & 0.00264 & 0.78465 \\
\hline $\mathrm{H}$ & 36 & 0.22612 & 0.00000 & 0.77226 & 0.00162 & 0.77388 \\
\hline $\mathrm{H}$ & 37 & 0.23747 & 0.00000 & 0.76135 & 0.00118 & 0.76253 \\
\hline $\mathrm{H}$ & 38 & 0.25129 & 0.00000 & 0.74696 & 0.00176 & 0.74871 \\
\hline $\mathrm{H}$ & 39 & 0.23418 & 0.00000 & 0.76447 & 0.00136 & 0.76582 \\
\hline $\mathrm{H}$ & 40 & 0.24938 & 0.00000 & 0.74927 & 0.00135 & 0.75062 \\
\hline $\mathrm{H}$ & 41 & 0.24188 & 0.00000 & 0.75696 & 0.00117 & 0.75812 \\
\hline $\mathrm{N}$ & 42 & -1.15517 & 1.99967 & 6.13928 & 0.01622 & 8.15517 \\
\hline $\mathrm{N}$ & 43 & -1.15199 & 1.99968 & 6.13583 & 0.01648 & 8.15199 \\
\hline $\mathrm{N}$ & 44 & -1.15369 & 1.99968 & 6.13761 & 0.01640 & 8.15369 \\
\hline $\mathrm{N}$ & 45 & -1.15656 & 1.99967 & 6.14059 & 0.01631 & 8.15656 \\
\hline $\mathrm{H}$ & 46 & 0.38572 & 0.00000 & 0.61295 & 0.00132 & 0.61428 \\
\hline $\mathrm{H}$ & 47 & 0.39168 & 0.00000 & 0.60621 & 0.00210 & 0.60832 \\
\hline $\mathrm{H}$ & 48 & 0.38997 & 0.00000 & 0.60789 & 0.00214 & 0.61003 \\
\hline $\mathrm{H}$ & 49 & 0.38812 & 0.00000 & 0.61046 & 0.00142 & 0.61188 \\
\hline $\mathrm{H}$ & 50 & 0.38695 & 0.00000 & 0.61185 & 0.00120 & 0.61305 \\
\hline $\mathrm{H}$ & 51 & 0.39278 & 0.00000 & 0.60518 & 0.00204 & 0.60722 \\
\hline $\mathrm{H}$ & 52 & 0.39008 & 0.00000 & 0.60836 & 0.00156 & 0.60992 \\
\hline $\mathrm{H}$ & 53 & 0.38598 & 0.00000 & 0.61277 & 0.00125 & 0.61402 \\
\hline $\mathrm{H}$ & 54 & 0.39405 & 0.00000 & 0.60409 & 0.00186 & 0.60595 \\
\hline $\mathrm{H}$ & 55 & 0.38963 & 0.00000 & 0.60860 & 0.00176 & 0.61037 \\
\hline $\mathrm{H}$ & 56 & 0.38357 & 0.00000 & 0.61498 & 0.00145 & 0.61643 \\
\hline $\mathrm{H}$ & 57 & 0.39302 & 0.00000 & 0.60412 & 0.00286 & 0.60698 \\
\hline Tot & Eal & 0.00000 & 115.98081 & 131.60754 & 0.41165 & 248.00000 \\
\hline
\end{tabular}

Natural Population 


\begin{tabular}{|c|c|c|c|c|}
\hline Effective Core & 66.00000 & & & \\
\hline Core & 49.98081 & ( $99.9616 \%$ & of & $50)$ \\
\hline Valence & 131.60754 & ( $99.7027 \%$ & of & $132)$ \\
\hline Natural Minimal Basis & 247.58835 & ( $99.8340 \%$ & of & $248)$ \\
\hline Natural Rydberg Basis & 0.41165 & $(0.1660 \%$ & of & $248)$ \\
\hline
\end{tabular}

Atom No Natural Electron Configuration

\begin{tabular}{|c|c|c|c|c|c|}
\hline $\mathrm{C}$ & 1 & [core] 2s ( & $0.96) 2 \mathrm{p}($ & $3.39) 3 p($ & $0.01)$ \\
\hline $\mathrm{C}$ & 2 & [core] 2s ( & $0.97) 2 \mathrm{p}($ & $3.43) 3 \mathrm{p}($ & $0.01)$ \\
\hline $\mathrm{C}$ & 3 & [core] 2s ( & $0.96) 2 \mathrm{p}($ & $3.40) 3 \mathrm{p}($ & $0.01)$ \\
\hline $\mathrm{C}$ & 4 & [core] 2s ( & $0.97) 2 \mathrm{p}($ & $3.40) 3 \mathrm{p}($ & $0.01)$ \\
\hline C & 5 & [core] 2s ( & $0.97) 2 \mathrm{p}($ & $3.42) 3 \mathrm{p}($ & $0.01)$ \\
\hline $\mathrm{Ga}$ & 6 & [core] 4s ( & $0.93) 4 \mathrm{p}($ & $1.03)$ & \\
\hline $\mathrm{N}$ & 7 & [core] 2s ( & $1.34) 2 \mathrm{p}($ & $4.46) 3 p($ & $0.01)$ \\
\hline C & 8 & [core] 2s ( & $1.08) 2 \mathrm{p}($ & $3.39) 3 \mathrm{p}($ & $0.01) 3 d(0.01)$ \\
\hline $\mathrm{Ga}$ & 9 & [core] 4s ( & $0.94) 4 \mathrm{p}($ & $1.03)$ & \\
\hline $\mathrm{N}$ & 10 & [core] 2s ( & $1.34) 2 \mathrm{p}($ & $4.46) 3 p($ & $0.01)$ \\
\hline $\mathrm{C}$ & 11 & [core] 2s ( & 1.08 ) 2p ( & $3.39) 3 \mathrm{p}($ & $0.01) 3 d(0.01)$ \\
\hline $\mathrm{N}$ & 12 & [core] 2s ( & $1.34) 2 \mathrm{p}($ & $4.46) 3 \mathrm{p}($ & $0.01)$ \\
\hline $\mathrm{C}$ & 13 & [core] 2s ( & $0.92) 2 \mathrm{p}($ & $3.19) 3 \mathrm{p}($ & 0.01 ) \\
\hline $\mathrm{C}$ & 14 & [core] 2s ( & $0.92) 2 \mathrm{p}($ & $3.19) 3 \mathrm{p}($ & $0.01)$ \\
\hline $\mathrm{N}$ & 15 & [core] 2s ( & $1.34) 2 \mathrm{p}($ & $4.46) 3 \mathrm{p}($ & $0.01)$ \\
\hline $\mathrm{C}$ & 16 & [core] 2s ( & $0.92) 2 \mathrm{p}($ & $3.19) 3 \mathrm{p}($ & $0.01)$ \\
\hline $\mathrm{C}$ & 17 & [core] 2s ( & $0.92) 2 \mathrm{p}($ & $3.19) 3 \mathrm{p}($ & $0.01)$ \\
\hline $\mathrm{C}$ & 18 & [core] 2s ( & $1.08) 2 \mathrm{p}($ & $3.39) 3 \mathrm{p}($ & $0.01) 3 d(0.01)$ \\
\hline $\mathrm{C}$ & 19 & [core] 2s ( & $1.08) 2 \mathrm{p}($ & $3.39) 3 \mathrm{p}($ & $0.01) 3 d(0.01)$ \\
\hline $\mathrm{K}$ & 20 & [core] 4s ( & $0.09) 4 \mathrm{p}($ & $0.01)$ & \\
\hline $\mathrm{H}$ & 21 & Is & $0.78)$ & & \\
\hline $\mathrm{H}$ & 22 & 1si & $0.78)$ & & \\
\hline $\mathrm{H}$ & 23 & 1s ( & $0.78)$ & & \\
\hline $\mathrm{H}$ & 24 & 1s ( & $0.78)$ & & \\
\hline $\mathrm{H}$ & 25 & 1s ( & $0.77)$ & & \\
\hline $\mathrm{H}$ & 26 & $1 \mathrm{~s}($ & $0.80)$ & & \\
\hline $\mathrm{H}$ & 27 & $1 \mathrm{si}$ & $0.78)$ & & \\
\hline $\mathrm{H}$ & 28 & 1s ( & $0.77)$ & & \\
\hline $\mathrm{H}$ & 29 & Is ( & $0.80)$ & & \\
\hline $\mathrm{H}$ & 30 & 1s ( & $0.78)$ & & \\
\hline $\mathrm{H}$ & 31 & 1si & $0.79)$ & & \\
\hline $\mathrm{H}$ & 32 & 1s ( & $0.78)$ & & \\
\hline $\mathrm{H}$ & 33 & 1s ( & $0.77)$ & & \\
\hline $\mathrm{H}$ & 34 & 1si & $0.80)$ & & \\
\hline $\mathrm{H}$ & 35 & 1s ( & $0.78)$ & & \\
\hline $\mathrm{H}$ & 36 & 1s ( & $0.77)$ & & \\
\hline $\mathrm{H}$ & 37 & 1s ( & $0.76)$ & & \\
\hline $\mathrm{H}$ & 38 & 1s ( & $0.75)$ & & \\
\hline $\mathrm{H}$ & 39 & 1s ( & $0.76)$ & & \\
\hline $\mathrm{H}$ & 40 & 1si & $0.75)$ & & \\
\hline $\mathrm{H}$ & 41 & $1 \mathrm{~s}($ & $0.76)$ & & \\
\hline $\mathrm{N}$ & 42 & [core] 2s ( & $1.54) 2 \mathrm{p}($ & $4.60) 3 \mathrm{p}($ & $0.01) 3 d(0.01)$ \\
\hline $\mathrm{N}$ & 43 & [core] 2s ( & $1.54) 2 \mathrm{p}($ & $4.60) 3 p($ & $0.01) 3 d(0.01)$ \\
\hline $\mathrm{N}$ & 44 & [core] 2s ( & $1.54) 2 \mathrm{p}($ & $4.60) 3 \mathrm{p}($ & $0.01) 3 d(0.01)$ \\
\hline $\mathrm{N}$ & 45 & [core] 2s ( & $1.54) 2 \mathrm{p}($ & $4.60) 3 \mathrm{p}($ & $0.01) 3 d(0.01)$ \\
\hline $\mathrm{H}$ & 46 & $1 \mathrm{~s}$ & $0.61)$ & & \\
\hline $\mathrm{H}$ & 47 & 1s ( & $0.61)$ & & \\
\hline $\mathrm{H}$ & 48 & 1s ( & $0.61)$ & & \\
\hline $\mathrm{H}$ & 49 & 1si & $0.61)$ & & \\
\hline $\mathrm{H}$ & 50 & 1s ( & $0.61)$ & & \\
\hline $\mathrm{H}$ & 51 & 1si & $0.61)$ & & \\
\hline
\end{tabular}


H 52

1s $(0.61)$

Is $(0.61)$

1s $(0.60)$

$1 \mathrm{~s}(0.61)$

Is $(0.61)$

Is $(0.60)$ 
Table 1. Crystal data for compound 2 CCDC No. 241279.

\begin{tabular}{|c|c|}
\hline Identification code & cj 301 \\
\hline Empirical formula & C69 H109 Ga2 K N8 \\
\hline Formula weight & 1229.18 \\
\hline Temperature & $150(2) \mathrm{K}$ \\
\hline Wavelength & $0.71073 \mathrm{~A}$ \\
\hline Crystal system, space group & monoclinic, $\quad \mathrm{C} 2 / \mathrm{C}$ \\
\hline Unit cell dimensions & $\begin{array}{l}\mathrm{a}=21.284(4) \text { A alpha }=90 \mathrm{deg} . \\
\mathrm{b}=15.538(3) \text { A beta }=114.31(3) \mathrm{deg} \\
\mathrm{c}=23.066(5) \text { A gamma }=90 \mathrm{deg} .\end{array}$ \\
\hline Volume & $6952(2) A^{\wedge} 3$ \\
\hline Z, Calculated density & 4, $1.174 \mathrm{Mg} / \mathrm{m}^{\wedge} 3$ \\
\hline Absorption coefficient & $0.879 \mathrm{~mm}^{\wedge}-1$ \\
\hline$F(0000)$ & 2640 \\
\hline Crystal size & $0.30 \times 0.15 \times 0.15 \mathrm{~mm}$ \\
\hline Theta range for data collection & 2.96 to $25.34 \mathrm{deg}$ \\
\hline Limiting indices & $-24<=\mathrm{h}<=23, \quad-16<=\mathrm{k}<=18, \quad-27<=1<=27$ \\
\hline Reflections collected / unique & $24083 / 6285[R($ int $)=0.0728]$ \\
\hline Completeness to theta $=25.34$ & $98.8 \%$ \\
\hline Max. and min. transmission & Sortav 0.851 and 0.654 \\
\hline Refinement method & Full-matrix least-squares on $\mathrm{F}^{\wedge} 2$ \\
\hline Data / restraints / parameters & $6285 / 46 / 397$ \\
\hline Goodness-of-fit on $F^{\wedge} 2$ & 1.032 \\
\hline Final R indices [I>2sigma(I)] & $\mathrm{R} 1=0.0505, \mathrm{wR} 2=0.1159$ \\
\hline R indices (all data) & $\mathrm{R} 1=0.0699, \quad \mathrm{wR} 2=0.1257$ \\
\hline Extinction coefficient & $0.0010(2)$ \\
\hline
\end{tabular}


Table 2. Atomic coordinates $\left(\mathrm{x}^{\left.10^{\wedge} 4\right)}\right.$ ) and equivalent isotropic displacement parameters $\left(A^{\wedge} 2 \times 10^{\wedge} 3\right)$ for $\mathrm{cj}^{3} 301$.

$\mathrm{U}(\mathrm{eq})$ is defined as one third of the trace of the orthogonalized Uij tensor.

\begin{tabular}{|c|c|c|c|c|}
\hline & $\mathrm{x}$ & $\mathrm{Y}$ & $z$ & $\mathrm{U}(\mathrm{eq})$ \\
\hline $\mathrm{Ga}(1)$ & 349 (1) & $8521(1)$ & $2191(1)$ & $26(1)$ \\
\hline $\mathrm{K}(1)$ & 0 & $5024(1)$ & 2500 & $49(1)$ \\
\hline $\mathrm{N}(1)$ & $147(1)$ & $9329(2)$ & $1516(1)$ & $29(1)$ \\
\hline $\mathrm{N}(2)$ & $1295(1)$ & $8599(2)$ & $2305(1)$ & $29(1)$ \\
\hline $\mathrm{N}(3)$ & $-592(2)$ & $4483(3)$ & $1096(2)$ & $72(1)$ \\
\hline $\mathrm{N}(4)$ & $899(2)$ & $4157(3)$ & $2088(2)$ & $72(1)$ \\
\hline$C(1)$ & $758(2)$ & $9572(2)$ & $1470(2)$ & $32(1)$ \\
\hline$C(2)$ & $1341(2)$ & $9200(2)$ & $1871(2)$ & $33(1)$ \\
\hline$C(3)$ & $-484(2)$ & $9539(2)$ & $979(2)$ & $31(1)$ \\
\hline$C(4)$ & $-666(2)$ & $9091(2)$ & $405(2)$ & $31(1)$ \\
\hline$C(5)$ & $-1251(2)$ & $9348(3)$ & $-118(2)$ & $40(1)$ \\
\hline$C(6)$ & $-1658(2)$ & $10018(3)$ & $-77(2)$ & $50(1)$ \\
\hline$C(7)$ & $-1483(2)$ & $10437(3)$ & $490(2)$ & $49(1)$ \\
\hline$C(8)$ & $-892(2)$ & $10221(2)$ & $1031(2)$ & $38(1)$ \\
\hline$C(9)$ & $-240(2)$ & $8337(2)$ & $344(2)$ & $32(1)$ \\
\hline$C(10)$ & $-653(2)$ & $7504(3)$ & $165(2)$ & $58(1)$ \\
\hline$C(11)$ & $85(3)$ & $8521(3)$ & $-115(2)$ & $67(1)$ \\
\hline$C(12)$ & $-680(2)$ & $10744(3)$ & $1630(2)$ & $49(1)$ \\
\hline$C(13)$ & $-1218(3)$ & $10781(3)$ & $1903(2)$ & $67(1)$ \\
\hline$C(14)$ & $-467(4)$ & $11636(4)$ & $1537(3)$ & $105(2)$ \\
\hline$C(15)$ & $1828(2)$ & $7973(2)$ & $2553(2)$ & $30(1)$ \\
\hline$C(16)$ & $2191(2)$ & $7882(2)$ & $3217(2)$ & $36(1)$ \\
\hline$C(17)$ & $2682(2)$ & $7235(3)$ & $3451(2)$ & $48(1)$ \\
\hline$C(18)$ & $2833(2)$ & $6687(3)$ & $3056(2)$ & $47(1)$ \\
\hline$C(19)$ & $2487(2)$ & $6785(3)$ & $2406(2)$ & $40(1)$ \\
\hline$C(20)$ & $1981(2)$ & $7414(2)$ & $2143(2)$ & $33(1)$ \\
\hline$C(21)$ & $2077(2)$ & $8501(3)$ & $3675(2)$ & $42(1)$ \\
\hline$C(22)$ & $2125(3)$ & $8073(4)$ & $4279(2)$ & $71(2)$ \\
\hline$C(23)$ & $2594(3)$ & $9238(4)$ & $3831(3)$ & $82(2)$ \\
\hline$C(24)$ & $1608(2)$ & $7462(3)$ & $1418(2)$ & $40(1)$ \\
\hline$C(25)$ & $1315(2)$ & $6597(3)$ & $1124(2)$ & $58(1)$ \\
\hline$C(26)$ & $2086(2)$ & $7811(3)$ & $1125(2)$ & $59(1)$ \\
\hline$C(27)$ & $-653(4)$ & $5238(4)$ & $721(3)$ & $119(3)$ \\
\hline$C(28)$ & $-1261(3)$ & $4066(6)$ & $865(4)$ & $123(3)$ \\
\hline$C(29)$ & $-92(3)$ & $3907(4)$ & $1046(3)$ & $86(2)$ \\
\hline$C(30)$ & $661(3)$ & $4120(5)$ & $1408(3)$ & $91(2)$ \\
\hline$C(31)$ & $1015(4)$ & $3319(5)$ & $2342(4)$ & $138(3)$ \\
\hline$C(32)$ & $1550(3)$ & $4615(5)$ & $2390(3)$ & $110(2)$ \\
\hline$C(33)$ & $184(10)$ & $6918(6)$ & $2066(7)$ & $33(2)$ \\
\hline$C(34)$ & $-517(7)$ & $6888(6)$ & $1982(8)$ & $41(2)$ \\
\hline$C(35)$ & $506(9)$ & $6855(7)$ & $2403(12)$ & $38(2)$ \\
\hline$C(36)$ & $-176(13)$ & $6836(7)$ & $1948(5)$ & $49(3)$ \\
\hline$C(37)$ & $-611(4)$ & $6850(6)$ & $2273(9)$ & $30(2)$ \\
\hline
\end{tabular}


Table 3. Bond lengths [A] and angles [deg] for cj301.

\begin{tabular}{|c|c|}
\hline $\mathrm{Ga}(1)-\mathrm{N}(1)$ & $1.907(3)$ \\
\hline $\mathrm{Ga}(1)-\mathrm{N}(2)$ & $1.924(3)$ \\
\hline $\mathrm{Ga}(1)-\mathrm{Ga}(1) \# 1$ & $2.4461(8)$ \\
\hline $\mathrm{K}(1)-\mathrm{N}(4) \# 1$ & $2.806(4)$ \\
\hline $\mathrm{K}(1)-\mathrm{N}(4)$ & $2.806(4)$ \\
\hline$K(1)-C(36) \# 1$ & $3.049(11)$ \\
\hline$K(1)-C(36)$ & $3.049(11)$ \\
\hline $\mathrm{K}(1)-\mathrm{N}(3)$ & $3.070(4)$ \\
\hline $\mathrm{K}(1)-\mathrm{N}(3) \# 1$ & $3.070(4)$ \\
\hline $\mathrm{K}(1)-\mathrm{C}(37) \# 1$ & $3.076(9)$ \\
\hline$K(1)-C(37)$ & $3.076(9)$ \\
\hline$K(1)-C(35)$ & $3.083(10)$ \\
\hline $\mathrm{K}(1)-\mathrm{C}(35) \# 1$ & $3.083(10)$ \\
\hline$K(1)-C(34) \# 1$ & $3.153(10)$ \\
\hline $\mathrm{K}(1)-\mathrm{C}(34)$ & $3.153(10)$ \\
\hline$N(1)-C(1)$ & $1.400(4)$ \\
\hline$N(1)-C(3)$ & $1.440(4)$ \\
\hline$N(2)-C(2)$ & $1.401(4)$ \\
\hline$N(2)-C(15)$ & $1.423(4)$ \\
\hline$N(3)-C(29)$ & $1.431(7)$ \\
\hline$N(3)-C(27)$ & $1.431(7)$ \\
\hline$N(3)-C(28)$ & $1.452(7)$ \\
\hline$N(4)-C(31)$ & $1.407(8)$ \\
\hline$N(4)-C(30)$ & $1.438(7)$ \\
\hline$N(4)-C(32)$ & $1.455(7)$ \\
\hline$C(1)-C(2)$ & $1.336(5)$ \\
\hline$C(3)-C(4)$ & $1.401(5)$ \\
\hline$C(3)-C(8)$ & $1.407(5)$ \\
\hline$C(4)-C(5)$ & $1.389(5)$ \\
\hline$C(4)-C(9)$ & $1.523(5)$ \\
\hline$C(5)-C(6)$ & $1.381(6)$ \\
\hline$C(6)-C(7)$ & $1.368(6)$ \\
\hline$C(7)-C(8)$ & $1.400(5)$ \\
\hline$C(8)-C(12)$ & $1.503(5)$ \\
\hline$C(9)-C(11)$ & $1.510(5)$ \\
\hline$C(9)-C(10)$ & $1.523(5)$ \\
\hline$C(12)-C(14)$ & $1.500(7)$ \\
\hline$C(12)-C(13)$ & $1.517(5)$ \\
\hline$C(15)-C(16)$ & $1.409(5)$ \\
\hline$C(15)-C(20)$ & $1.418(5)$ \\
\hline$C(16)-C(17)$ & $1.389(5)$ \\
\hline$C(16)-C(21)$ & $1.519(5)$ \\
\hline$C(17)-C(18)$ & $1.378(5)$ \\
\hline$C(18)-C(19)$ & $1.380(5)$ \\
\hline$C(19)-C(20)$ & $1.394(5)$ \\
\hline$C(20)-C(24)$ & $1.529(5)$ \\
\hline$C(21)-C(22)$ & $1.509(6)$ \\
\hline$C(21)-C(23)$ & $1.524(6)$ \\
\hline$C(24)-C(25)$ & $1.519(6)$ \\
\hline$C(24)-C(26)$ & $1.535(5)$ \\
\hline$C(29)-C(30)$ & $1.508(7)$ \\
\hline$C(33)-C(37) \# 1$ & $1.422(9)$ \\
\hline$C(33)-C(34)$ & $1.423(10)$ \\
\hline$C(33)-C(37)$ & $1.941(12)$ \\
\hline$C(33)-C(34) \# 1$ & $2.011(12)$ \\
\hline$C(34)-C(35) \# 1$ & $1.409(10)$ \\
\hline$C(34)-C(35)$ & $1.985(13)$ \\
\hline$C(34)-C(33) \# 1$ & $2.011(12)$ \\
\hline
\end{tabular}




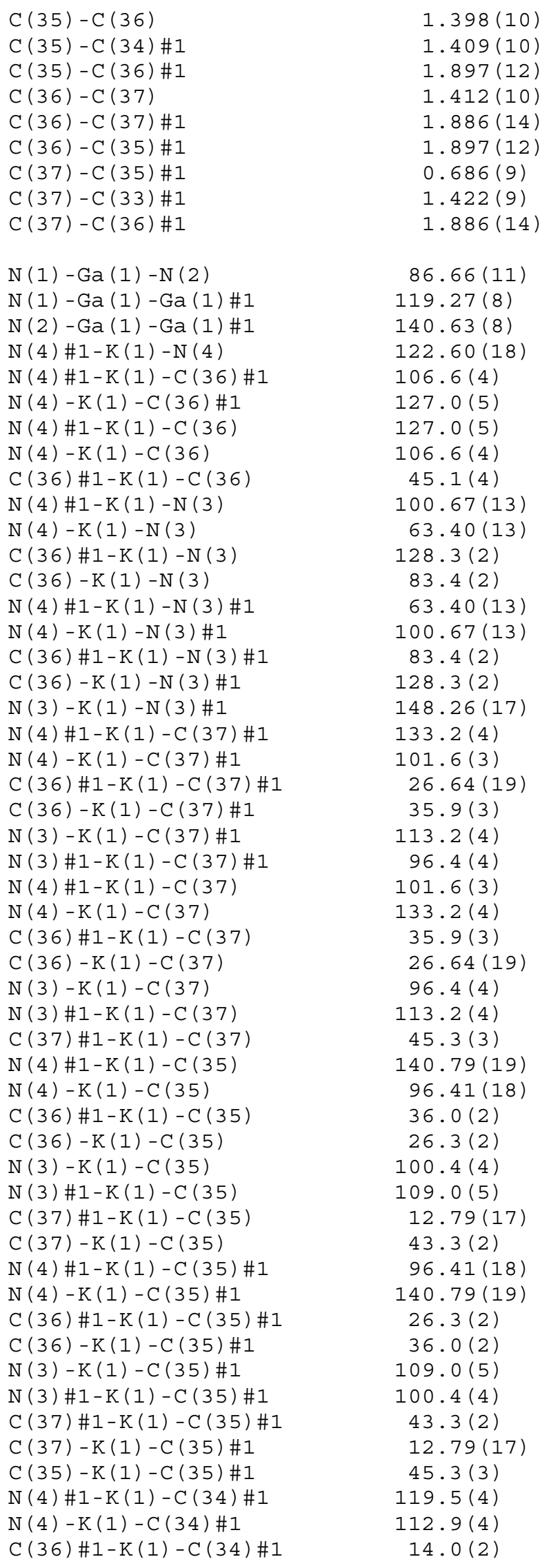

$1.398(10)$
$1.409(10)$
$1.897(12)$
$1.412(10)$
$1.886(14)$
$1.897(12)$
$0.686(9)$
$1.422(9)$
$1.886(14)$

$86.66(11)$

$119.27(8)$

$140.63(8)$

$122.60(18)$

$106.6(4)$

$127.0(5)$

$127.0(5)$

$106.6(4)$

$45.1(4)$

$100.67(13)$

$63.40(13)$

$128.3(2)$

$83.4(2)$

$63.40(13)$

$100.67(13)$

$83.4(2)$

$128.3(2)$

$148.26(17)$

$133.2(4)$

$101.6(3)$

26.64 (19)

$35.9(3)$

$113.2(4)$

$96.4(4)$

$101.6(3)$

$133.2(4)$

$35.9(3)$

26.64 (19)

$96.4(4)$

$113.2(4)$

$45.3(3)$

140.79 (19)

$96.41(18)$

$36.0(2)$

$26.3(2)$

$100.4(4)$

$109.0(5)$

$12.79(17)$

$43.3(2)$

$96.41(18)$

140.79 (19)

$26.3(2)$

$36.0(2)$

$109.0(5)$

$100.4(4)$

$43.3(2)$

$12.79(17)$

$45.3(3)$

$119.5(4)$

$112.9(4)$

$14.0(2)$ 


\begin{tabular}{|c|c|}
\hline$C(36)-K(1)-C(34) \# 1$ & $43.4(2)$ \\
\hline$N(3)-K(1)-C(34) \# 1$ & $125.4(3)$ \\
\hline $\mathrm{N}(3) \# 1-\mathrm{K}(1)-\mathrm{C}(34) \# 1$ & $85.6(3)$ \\
\hline $\mathrm{C}(37) \# 1-\mathrm{K}(1)-\mathrm{C}(34) \# 1$ & $14.27(16)$ \\
\hline $\mathrm{C}(37)-\mathrm{K}(1)-\mathrm{C}(34) \# 1$ & $43.5(2)$ \\
\hline$C(35)-K(1)-C(34) \# 1$ & $26.09(18)$ \\
\hline$C(35) \# 1-K(1)-C(34) \# 1$ & $37.1(3)$ \\
\hline $\mathrm{N}(4) \# 1-\mathrm{K}(1)-\mathrm{C}(34)$ & $112.9(4)$ \\
\hline$N(4)-K(1)-C(34)$ & $119.5(4)$ \\
\hline$C(36) \# 1-K(1)-C(34)$ & $43.4(2)$ \\
\hline$C(36)-K(1)-C(34)$ & $14.0(2)$ \\
\hline$N(3)-K(1)-C(34)$ & $85.6(3)$ \\
\hline $\mathrm{N}(3) \# 1-\mathrm{K}(1)-\mathrm{C}(34)$ & $125.4(3)$ \\
\hline$C(37) \# 1-K(1)-C(34)$ & $43.5(2)$ \\
\hline$C(37)-K(1)-C(34)$ & $14.27(16)$ \\
\hline$C(35)-K(1)-C(34)$ & $37.1(3)$ \\
\hline$C(35) \# 1-K(1)-C(34)$ & $26.09(18)$ \\
\hline $\mathrm{C}(34) \# 1-\mathrm{K}(1)-\mathrm{C}(34)$ & $46.6(4)$ \\
\hline$C(1)-N(1)-C(3)$ & $116.2(3)$ \\
\hline $\mathrm{C}(1)-\mathrm{N}(1)-\mathrm{Ga}(1)$ & $109.5(2)$ \\
\hline $\mathrm{C}(3)-\mathrm{N}(1)-\mathrm{Ga}(1)$ & $131.9(2)$ \\
\hline$C(2)-N(2)-C(15)$ & $117.7(3)$ \\
\hline $\mathrm{C}(2)-\mathrm{N}(2)-\mathrm{Ga}(1)$ & $108.8(2)$ \\
\hline $\mathrm{C}(15)-\mathrm{N}(2)-\mathrm{Ga}(1)$ & $128.9(2)$ \\
\hline $\mathrm{C}(29)-\mathrm{N}(3)-\mathrm{C}(27)$ & $110.5(5)$ \\
\hline$C(29)-N(3)-C(28)$ & $109.9(5)$ \\
\hline$C(27)-N(3)-C(28)$ & $108.3(5)$ \\
\hline$C(29)-N(3)-K(1)$ & $105.0(3)$ \\
\hline $\mathrm{C}(27)-\mathrm{N}(3)-\mathrm{K}(1)$ & $108.0(3)$ \\
\hline $\mathrm{C}(28)-\mathrm{N}(3)-\mathrm{K}(1)$ & $115.0(4)$ \\
\hline$C(31)-N(4)-C(30)$ & $109.9(6)$ \\
\hline$C(31)-N(4)-C(32)$ & $106.4(6)$ \\
\hline$C(30)-N(4)-C(32)$ & $112.2(5)$ \\
\hline $\mathrm{C}(31)-\mathrm{N}(4)-\mathrm{K}(1)$ & $109.3(4)$ \\
\hline $\mathrm{C}(30)-\mathrm{N}(4)-\mathrm{K}(1)$ & $114.0(3)$ \\
\hline $\mathrm{C}(32)-\mathrm{N}(4)-\mathrm{K}(1)$ & $104.6(4)$ \\
\hline$C(2)-C(1)-N(1)$ & $117.3(3)$ \\
\hline$C(1)-C(2)-N(2)$ & $117.7(3)$ \\
\hline$C(4)-C(3)-C(8)$ & $121.0(3)$ \\
\hline$C(4)-C(3)-N(1)$ & $119.8(3)$ \\
\hline$C(8)-C(3)-N(1)$ & $119.1(3)$ \\
\hline$C(5)-C(4)-C(3)$ & $118.5(3)$ \\
\hline$C(5)-C(4)-C(9)$ & $119.7(3)$ \\
\hline$C(3)-C(4)-C(9)$ & $121.7(3)$ \\
\hline$C(6)-C(5)-C(4)$ & $121.3(4)$ \\
\hline$C(7)-C(6)-C(5)$ & $119.6(3)$ \\
\hline$C(6)-C(7)-C(8)$ & $121.9(4)$ \\
\hline$C(7)-C(8)-C(3)$ & $117.6(3)$ \\
\hline$C(7)-C(8)-C(12)$ & $120.7(3)$ \\
\hline$C(3)-C(8)-C(12)$ & $121.6(3)$ \\
\hline$C(11)-C(9)-C(4)$ & $112.4(3)$ \\
\hline$C(11)-C(9)-C(10)$ & $110.5(3)$ \\
\hline$C(4)-C(9)-C(10)$ & $112.3(3)$ \\
\hline$C(14)-C(12)-C(8)$ & $111.3(4)$ \\
\hline$C(14)-C(12)-C(13)$ & $110.3(4)$ \\
\hline$C(8)-C(12)-C(13)$ & $114.1(4)$ \\
\hline$C(16)-C(15)-C(20)$ & $119.3(3)$ \\
\hline $\mathrm{C}(16)-\mathrm{C}(15)-\mathrm{N}(2)$ & $119.7(3)$ \\
\hline $\mathrm{C}(20)-\mathrm{C}(15)-\mathrm{N}(2)$ & $120.9(3)$ \\
\hline$C(17)-C(16)-C(15)$ & $118.9(3)$ \\
\hline$C(17)-C(16)-C(21)$ & $119.8(3)$ \\
\hline
\end{tabular}




\begin{tabular}{|c|c|}
\hline$C(15)-C(16)-C(21)$ & $121.2(3)$ \\
\hline$C(18)-C(17)-C(16)$ & $122.3(3)$ \\
\hline$C(17)-C(18)-C(19)$ & $118.9(3)$ \\
\hline$C(18)-C(19)-C(20)$ & $121.4(3)$ \\
\hline$C(19)-C(20)-C(15)$ & $119.2(3)$ \\
\hline$C(19)-C(20)-C(24)$ & $118.1(3)$ \\
\hline$C(15)-C(20)-C(24)$ & $122.7(3)$ \\
\hline$C(22)-C(21)-C(16)$ & $113.2(4)$ \\
\hline$C(22)-C(21)-C(23)$ & $110.2(4)$ \\
\hline$C(16)-C(21)-C(23)$ & $109.6(3)$ \\
\hline$C(25)-C(24)-C(20)$ & $112.2(3)$ \\
\hline$C(25)-C(24)-C(26)$ & $109.9(3)$ \\
\hline$C(20)-C(24)-C(26)$ & $111.1(3)$ \\
\hline$N(3)-C(29)-C(30)$ & $118.3(5)$ \\
\hline$N(4)-C(30)-C(29)$ & $115.4(5)$ \\
\hline$C(37) \# 1-C(33)-C(34)$ & $108.4(7)$ \\
\hline$C(37) \# 1-C(33)-C(37)$ & $88.3(6)$ \\
\hline$C(34)-C(33)-C(37)$ & $20.1(4)$ \\
\hline$C(37) \# 1-C(33)-C(34) \# 1$ & $17.3(4)$ \\
\hline$C(34)-C(33)-C(34) \# 1$ & $91.5(7)$ \\
\hline$C(37)-C(33)-C(34) \# 1$ & $71.4(5)$ \\
\hline$C(37) \# 1-C(33)-K(1)$ & $72.7(5)$ \\
\hline$C(34)-C(33)-K(1)$ & $75.8(6)$ \\
\hline$C(37)-C(33)-K(1)$ & $68.9(4)$ \\
\hline$C(34) \# 1-C(33)-K(1)$ & $70.7(4)$ \\
\hline$C(35) \# 1-C(34)-C(33)$ & $106.4(8)$ \\
\hline$C(35) \# 1-C(34)-C(35)$ & $86.9(7)$ \\
\hline$C(33)-C(34)-C(35)$ & $19.6(4)$ \\
\hline$C(35) \# 1-C(34)-C(33) \# 1$ & $18.2(5)$ \\
\hline$C(33)-C(34)-C(33) \# 1$ & $88.4(7)$ \\
\hline$C(35)-C(34)-C(33) \# 1$ & $69.1(5)$ \\
\hline$C(35) \# 1-C(34)-K(1)$ & $74.2(6)$ \\
\hline$C(33)-C(34)-K(1)$ & $78.2(6)$ \\
\hline$C(35)-C(34)-K(1)$ & $69.5(5)$ \\
\hline$C(33) \# 1-C(34)-K(1)$ & $72.3(4)$ \\
\hline$C(36)-C(35)-C(34) \# 1$ & $109.8(7)$ \\
\hline$C(36)-C(35)-C(36) \# 1$ & $89.2(7)$ \\
\hline$C(34) \# 1-C(35)-C(36) \# 1$ & $20.8(5)$ \\
\hline$C(36)-C(35)-C(34)$ & $16.9(4)$ \\
\hline$C(34) \# 1-C(35)-C(34)$ & $93.0(7)$ \\
\hline$C(36) \# 1-C(35)-C(34)$ & $72.5(5)$ \\
\hline$C(36)-C(35)-K(1)$ & $75.5(6)$ \\
\hline$C(34) \# 1-C(35)-K(1)$ & $79.7(6)$ \\
\hline$C(36) \# 1-C(35)-K(1)$ & $71.0(5)$ \\
\hline$C(34)-C(35)-K(1)$ & $73.4(4)$ \\
\hline$C(35)-C(36)-C(37)$ & $107.8(7)$ \\
\hline$C(35)-C(36)-C(37) \# 1$ & $17.1(4)$ \\
\hline$C(37)-C(36)-C(37) \# 1$ & $90.8(7)$ \\
\hline$C(35)-C(36)-C(35) \# 1$ & $90.8(7)$ \\
\hline$C(37)-C(36)-C(35) \# 1$ & $17.0(4)$ \\
\hline$C(37) \# 1-C(36)-C(35) \# 1$ & $73.8(5)$ \\
\hline$C(35)-C(36)-K(1)$ & $78.2(6)$ \\
\hline$C(37)-C(36)-K(1)$ & $77.7(6)$ \\
\hline$C(37) \# 1-C(36)-K(1)$ & $72.8(5)$ \\
\hline$C(35) \# 1-C(36)-K(1)$ & $72.9(5)$ \\
\hline$C(35) \# 1-C(37)-C(36)$ & $125.9(13)$ \\
\hline$C(35) \# 1-C(37)-C(33) \# 1$ & $18.8(11)$ \\
\hline$C(36)-C(37)-C(33) \# 1$ & $107.5(7)$ \\
\hline$C(35) \# 1-C(37)-C(36) \# 1$ & $36.7(10)$ \\
\hline$C(36)-C(37)-C(36) \# 1$ & $89.2(7)$ \\
\hline$C(33) \# 1-C(37)-C(36) \# 1$ & $18.9(4)$ \\
\hline
\end{tabular}




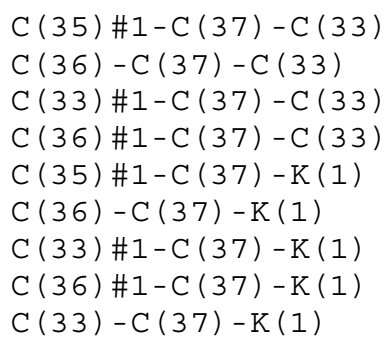

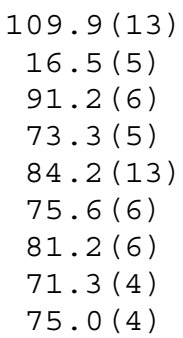

Symmetry transformations used to generate equivalent atoms: $\# 1-\mathrm{x}, \mathrm{y},-\mathrm{z}+1 / 2$

Table 4. Anisotropic displacement parameters (A^2 x 10^3) for $\mathrm{Cj}^{\wedge} 301$. The anisotropic displacement factor exponent takes the form: $-2 \mathrm{pi}^{\wedge} 2\left[\mathrm{~h}^{\wedge} 2 \mathrm{a}^{\star \wedge} 2 \mathrm{U} 11+\ldots+2 \mathrm{~h} k \mathrm{a}\right.$ * b* U12]

\begin{tabular}{|c|c|c|c|c|c|c|}
\hline & U11 & U2 2 & U3 3 & $\mathrm{U} 23$ & U13 & U12 \\
\hline $\mathrm{Ga}(1)$ & $22(1)$ & $39(1)$ & $21(1)$ & $0(1)$ & $12(1)$ & $1(1)$ \\
\hline K ( I ) & $49(1)$ & $34(1)$ & $77(1)$ & 0 & $38(1)$ & 0 \\
\hline $\mathrm{N}(1)$ & $24(1)$ & $41(2)$ & $24(1)$ & $3(1)$ & $13(1)$ & $2(1)$ \\
\hline $\mathrm{N}(2)$ & $24(1)$ & $37(2)$ & 27 (1) & $1(1)$ & $13(1)$ & $3(1)$ \\
\hline $\mathrm{N}(3)$ & $75(3)$ & $63(3)$ & $68(3)$ & $-1(2)$ & $20(2)$ & $-3(2)$ \\
\hline $\mathrm{N}(4)$ & $68(3)$ & $70(3)$ & $89(3)$ & $5(2)$ & $43(2)$ & $-1(2)$ \\
\hline$C(1)$ & $31(2)$ & $37(2)$ & $33(2)$ & $1(2)$ & $19(2)$ & $-2(2)$ \\
\hline$C(2)$ & $27(2)$ & $38(2)$ & $39(2)$ & $-1(2)$ & $19(2)$ & $-3(2)$ \\
\hline$C(3)$ & $27(2)$ & $40(2)$ & $29(2)$ & $9(2)$ & $16(1)$ & $3(1)$ \\
\hline$C(4)$ & $25(2)$ & 41 ( 2 ) & $28(2)$ & $10(2)$ & $13(1)$ & $0(1)$ \\
\hline$C(5)$ & $34(2)$ & $55(2)$ & $28(2)$ & $12(2)$ & $12(2)$ & $1(2)$ \\
\hline$C(6)$ & $34(2)$ & 75 (3) & $42(2)$ & $27(2)$ & $17(2)$ & $15(2)$ \\
\hline$C(7)$ & $43(2)$ & $56(3)$ & $58(3)$ & $24(2)$ & $31(2)$ & $21(2)$ \\
\hline$C(8)$ & $37(2)$ & $45(2)$ & $43(2)$ & $10(2)$ & $27(2)$ & $10(2)$ \\
\hline$C(9)$ & $30(2)$ & $42(2)$ & $24(2)$ & $0(1)$ & $11(1)$ & $0(2)$ \\
\hline$C(10)$ & $44(2)$ & $49(3)$ & $76(3)$ & $4(2)$ & $19(2)$ & $0(2)$ \\
\hline$C(11)$ & $98(4)$ & $56(3)$ & $80(3)$ & $16(2)$ & $72(3)$ & $20(3)$ \\
\hline$C(12)$ & $58(2)$ & $47(2)$ & $57(2)$ & $2(2)$ & $40(2)$ & $11(2)$ \\
\hline$C(13)$ & 79 (3) & $81(4)$ & $67(3)$ & $8(3)$ & $55(3)$ & $18(3)$ \\
\hline$C(14)$ & $161(5)$ & $99(4)$ & $92(4)$ & $-30(3)$ & $88(4)$ & $-40(4)$ \\
\hline$C(15)$ & $21(2)$ & $40(2)$ & $31(2)$ & $2(2)$ & $14(1)$ & $0(1)$ \\
\hline$C(16)$ & $26(2)$ & $50(2)$ & $32(2)$ & $-1(2)$ & $12(1)$ & $3(2)$ \\
\hline$C(17)$ & $39(2)$ & $69(3)$ & $31(2)$ & $3(2)$ & $9(2)$ & $12(2)$ \\
\hline$C(18)$ & $38(2)$ & $55(3)$ & $43(2)$ & $8(2)$ & $11(2)$ & $18(2)$ \\
\hline$C(19)$ & $33(2)$ & 49 (2) & $40(2)$ & $-1(2)$ & 19 (2) & $10(2)$ \\
\hline$C(20)$ & $24(2)$ & $46(2)$ & $32(2)$ & $0(2)$ & $13(1)$ & $3(2)$ \\
\hline$C(21)$ & $29(2)$ & $64(3)$ & $31(2)$ & $-8(2)$ & $11(2)$ & $3(2)$ \\
\hline$C(22)$ & $75(3)$ & $104(4)$ & $41(2)$ & $8(3)$ & $32(2)$ & $30(3)$ \\
\hline$C(23)$ & $68(3)$ & $97(4)$ & $99(4)$ & $-53(3)$ & $54(3)$ & $-26(3)$ \\
\hline$C(24)$ & $37(2)$ & $53(2)$ & $32(2)$ & $1(2)$ & $16(2)$ & $14(2)$ \\
\hline$C(25)$ & $55(2)$ & 75 (3) & $39(2)$ & $-12(2)$ & $13(2)$ & $10(2)$ \\
\hline$C(26)$ & $65(3)$ & 77 (3) & $48(2)$ & $12(2)$ & $35(2)$ & $13(2)$ \\
\hline$C(27)$ & $155(7)$ & $82(5)$ & $85(5)$ & $6(4)$ & $15(4)$ & $0(4)$ \\
\hline$C(28)$ & $69(4)$ & $164(8)$ & $123(6)$ & $-11(5)$ & $27(4)$ & $-4(4)$ \\
\hline$C(29)$ & $84(4)$ & $84(4)$ & $99(4)$ & $-9(3)$ & $48(3)$ & $-3(3)$ \\
\hline$C(30)$ & $78(4)$ & $124(5)$ & $84(4)$ & $-30(4)$ & $47(3)$ & $-16(4)$ \\
\hline$C(31)$ & $168(8)$ & $88(5)$ & $139(7)$ & $32(5)$ & $43(6)$ & $41(5)$ \\
\hline
\end{tabular}




\begin{tabular}{|c|c|c|c|c|c|c|}
\hline C (32) & $61(3)$ & $168(7)$ & $98(5)$ & $-2(5)$ & $30(3)$ & $-15(4)$ \\
\hline$C(33)$ & $32(5)$ & $36(4)$ & $31(5)$ & $-5(3)$ & $15(4)$ & $2(4)$ \\
\hline$C(34)$ & $38(5)$ & $32(4)$ & $48(5)$ & $-7(4)$ & $12(4)$ & $-6(4)$ \\
\hline$C(35)$ & $39(5)$ & $38(4)$ & $46(5)$ & $-10(5)$ & $27(5)$ & $-3(4)$ \\
\hline$C(36)$ & $63(6)$ & $41(5)$ & $44(5)$ & $-10(3)$ & $24(5)$ & $-1(5)$ \\
\hline$C(37)$ & $32(4)$ & $21(4)$ & $29(5)$ & $-8(4)$ & $3(4)$ & $-1(3)$ \\
\hline
\end{tabular}

Table 5. Hydrogen coordinates $\left(\mathrm{x}^{\left.10^{\wedge} 4\right)}\right.$ and isotropic displacement parameters $\left(\mathrm{A}^{\wedge} 2 \times 10^{\wedge} 3\right)$ for $\mathrm{Cj} 301$.

\begin{tabular}{|c|c|c|c|c|}
\hline & $\mathrm{x}$ & $\mathrm{Y}$ & $\mathrm{z}$ & $\mathrm{U}(\mathrm{eq})$ \\
\hline $\mathrm{H}(1)$ & 759 & 9985 & 1166 & 38 \\
\hline $\mathrm{H}(2)$ & 1772 & 9338 & 1863 & 39 \\
\hline $\mathrm{H}(5)$ & -1375 & 9057 & -512 & 47 \\
\hline $\mathrm{H}(6)$ & -2055 & 10186 & -440 & 60 \\
\hline $\mathrm{H}(7)$ & -1772 & 10888 & 517 & 58 \\
\hline $\mathrm{H}(9)$ & 144 & 8246 & 772 & 39 \\
\hline $\mathrm{H}(10 \mathrm{~A})$ & -1010 & 7550 & -270 & 87 \\
\hline $\mathrm{H}(10 \mathrm{~B})$ & -871 & 7403 & 459 & 87 \\
\hline $\mathrm{H}(10 \mathrm{C})$ & -345 & 7024 & 189 & 87 \\
\hline $\mathrm{H}(11 \mathrm{~A})$ & -276 & 8557 & -548 & 100 \\
\hline $\mathrm{H}(11 \mathrm{~B})$ & 406 & 8057 & -91 & 100 \\
\hline $\mathrm{H}(11 \mathrm{C})$ & 335 & 9068 & -2 & 100 \\
\hline $\mathrm{H}(12)$ & -263 & 10459 & 1957 & 58 \\
\hline $\mathrm{H}(13 \mathrm{~A})$ & -1645 & 11027 & 1586 & 101 \\
\hline $\mathrm{H}(13 \mathrm{~B})$ & -1048 & 11142 & 2285 & 101 \\
\hline $\mathrm{H}(13 \mathrm{C})$ & -1307 & 10198 & 2013 & 101 \\
\hline $\mathrm{H}(14 \mathrm{~A})$ & -69 & 11604 & 1428 & 158 \\
\hline $\mathrm{H}(14 \mathrm{~B})$ & -343 & 11966 & 1931 & 158 \\
\hline $\mathrm{H}(14 \mathrm{C})$ & -851 & 11920 & 1192 & 158 \\
\hline $\mathrm{H}(17)$ & 2921 & 7168 & 3898 & 57 \\
\hline $\mathrm{H}(18)$ & 3171 & 6250 & 3229 & 57 \\
\hline $\mathrm{H}(19)$ & 2595 & 6416 & 2132 & 48 \\
\hline $\mathrm{H}(21)$ & 1604 & 8749 & 3456 & 50 \\
\hline $\mathrm{H}(22 \mathrm{~A})$ & 1834 & 7557 & 4173 & 106 \\
\hline $\mathrm{H}(22 \mathrm{~B})$ & 1968 & 8474 & 4519 & 106 \\
\hline $\mathrm{H}(22 \mathrm{C})$ & 2605 & 7910 & 4538 & 106 \\
\hline $\mathrm{H}(23 \mathrm{~A})$ & 3063 & 9010 & 4049 & 123 \\
\hline $\mathrm{H}(23 \mathrm{~B})$ & 2504 & 9653 & 4108 & 123 \\
\hline $\mathrm{H}(23 \mathrm{C})$ & 2547 & 9524 & 3437 & 123 \\
\hline $\mathrm{H}(24)$ & 1214 & 7873 & 1311 & 48 \\
\hline $\mathrm{H}(25 \mathrm{~A})$ & 1693 & 6204 & 1173 & 87 \\
\hline $\mathrm{H}(25 \mathrm{~B})$ & 1021 & 6674 & 670 & 87 \\
\hline $\mathrm{H}(25 \mathrm{C})$ & 1041 & 6355 & 1337 & 87 \\
\hline $\mathrm{H}(26 \mathrm{~A})$ & 2272 & 8370 & 1316 & 89 \\
\hline $\mathrm{H}(26 \mathrm{~B})$ & 1825 & 7881 & 665 & 89 \\
\hline $\mathrm{H}(26 \mathrm{C})$ & 2466 & 7406 & 1206 & 89 \\
\hline $\mathrm{H}(27 \mathrm{~A})$ & -218 & 5560 & 896 & 178 \\
\hline $\mathrm{H}(27 \mathrm{~B})$ & -1026 & 5600 & 727 & 178 \\
\hline $\mathrm{H}(27 \mathrm{C})$ & -755 & 5070 & 281 & 178 \\
\hline $\mathrm{H}(28 \mathrm{~A})$ & -1600 & 4464 & 902 & 184 \\
\hline $\mathrm{H}(28 \mathrm{~B})$ & -1230 & 3550 & 1120 & 184 \\
\hline $\mathrm{H}(28 \mathrm{C})$ & -1403 & 3902 & 419 & 184 \\
\hline $\mathrm{H}(29 \mathrm{~A})$ & -184 & 3858 & 590 & 103 \\
\hline $\mathrm{H}(29 \mathrm{~B})$ & -172 & 3332 & 1187 & 103 \\
\hline
\end{tabular}




$\begin{array}{lrlll}\text { H (3OA) } & 937 & 3683 & 1303 & 109 \\ \mathrm{H}(30 \mathrm{~B}) & 752 & 4684 & 1257 & 109 \\ \mathrm{H}(31 \mathrm{~A}) & 580 & 2999 & 2180 & 208 \\ \mathrm{H}(31 \mathrm{~B}) & 1200 & 3350 & 2808 & 208 \\ \mathrm{H}(31 \mathrm{C}) & 1348 & 3025 & 2217 & 208 \\ \mathrm{H}(32 \mathrm{~A}) & 1661 & 4692 & 2843 & 164 \\ \mathrm{H}(32 \mathrm{~B}) & 1511 & 5179 & 2188 & 164 \\ \mathrm{H}(32 \mathrm{C}) & 1916 & 4282 & 2341 & 164\end{array}$




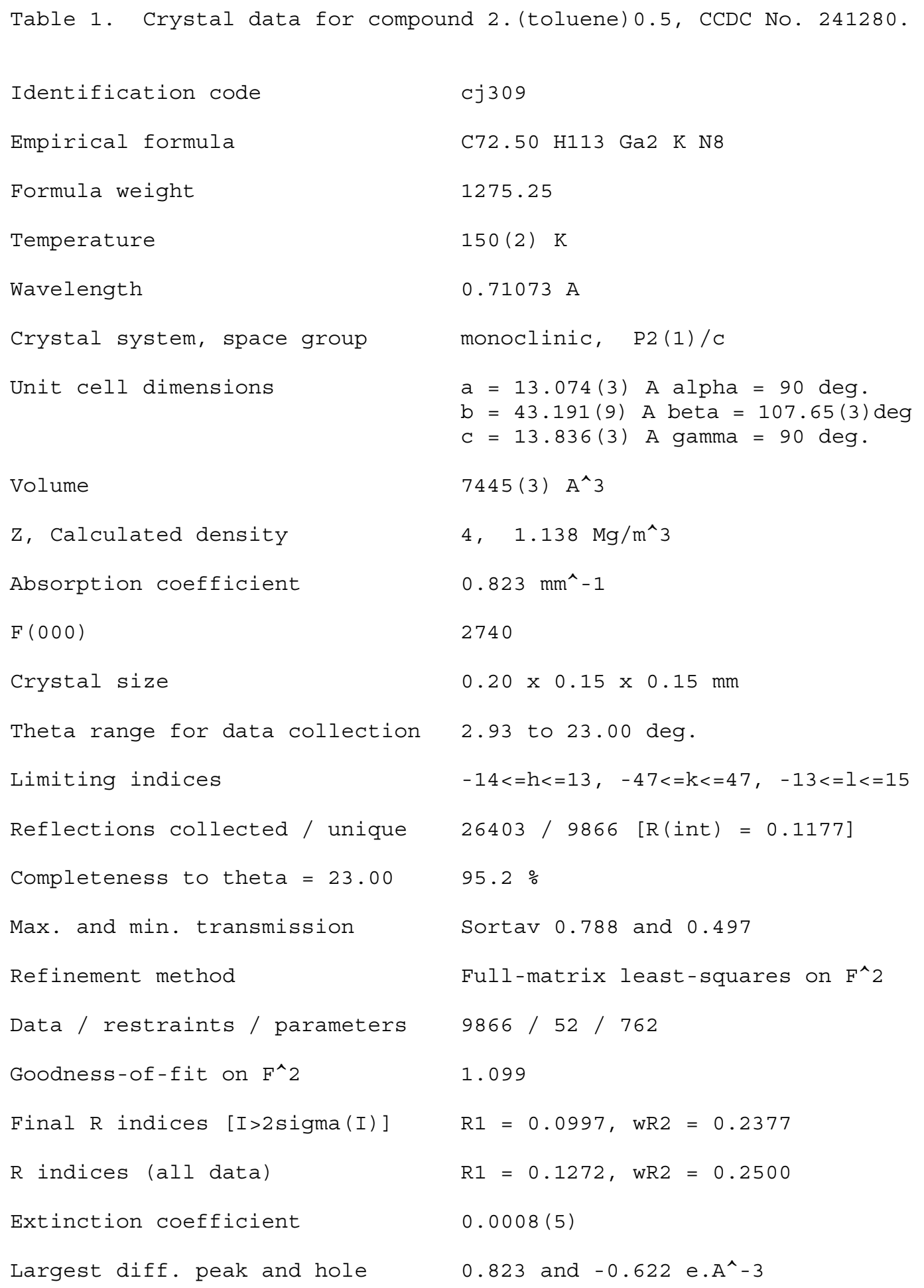


Table 2. Atomic coordinates $\left(\mathrm{x} 10^{\wedge} 4\right)$ and equivalent isotropic displacement parameters $\left(A^{\wedge} 2 \times 10^{\wedge} 3\right)$ for $\mathrm{Cj}^{3} 39$.

$\mathrm{U}(\mathrm{eq})$ is defined as one third of the trace of the orthogonalized Uij tensor.

\begin{tabular}{|c|c|c|c|c|}
\hline & $\mathrm{x}$ & $\mathrm{Y}$ & $z$ & $\mathrm{U}(\mathrm{eq})$ \\
\hline $\mathrm{Ga}(1)$ & $3933(1)$ & $1531(1)$ & $2970(1)$ & $31(1)$ \\
\hline $\mathrm{Ga}(2)$ & $5149(1)$ & $1206(1)$ & $2362(1)$ & $31(1)$ \\
\hline $\mathrm{K}(1)$ & $1041(2)$ & $1311(1)$ & $-841(2)$ & $51(1)$ \\
\hline $\mathrm{N}(1)$ & $3276(5)$ & $1501(1)$ & $4026(5)$ & $33(2)$ \\
\hline $\mathrm{N}(2)$ & $4491(5)$ & $1921(2)$ & $3544(5)$ & $34(2)$ \\
\hline $\mathrm{N}(3)$ & $6029(6)$ & $1242(2)$ & $1489(5)$ & $36(2)$ \\
\hline $\mathrm{N}(4)$ & $6244(5)$ & $946(2)$ & $3204(5)$ & $35(2)$ \\
\hline $\mathrm{N}(5)$ & $-548(7)$ & $1810(2)$ & $-1253(7)$ & $62(2)$ \\
\hline $\mathrm{N}(6)$ & $-845(7)$ & $1197(2)$ & $-356(9)$ & $72(3)$ \\
\hline $\mathrm{N}(7)$ & $1019(13)$ & $762(4)$ & $-2019(14)$ & $132(5)$ \\
\hline $\mathrm{N}(8)$ & $1602(10)$ & $1377(3)$ & $-2606(9)$ & $102(4)$ \\
\hline$C(1)$ & $3548(6)$ & $1761(2)$ & $4660(6)$ & $35(2)$ \\
\hline$C(2)$ & $4188(6)$ & $1971(2)$ & $4412(6)$ & $35(2)$ \\
\hline$C(3)$ & $2308(6)$ & $1342(2)$ & $4022(7)$ & $36(2)$ \\
\hline$C(4)$ & $2350(7)$ & $1025(2)$ & $4281(7)$ & $44(2)$ \\
\hline$C(5)$ & $1429(8)$ & $867(2)$ & $4279(9)$ & $57(3)$ \\
\hline$C(6)$ & $443(8)$ & $1021(3)$ & $3993(9)$ & $62(3)$ \\
\hline$C(7)$ & $385(7)$ & $1325(2)$ & $3762(8)$ & $51(3)$ \\
\hline$C(8)$ & $1294(7)$ & $1498(2)$ & $3738(7)$ & $43(2)$ \\
\hline$C(9)$ & $3428(7)$ & $853(2)$ & $4603(8)$ & $46(2)$ \\
\hline$C(10)$ & $3739(9)$ & $770(3)$ & $5724(9)$ & $74(3)$ \\
\hline$C(11)$ & $3376(9)$ & $563(2)$ & $3989(9)$ & $66(3)$ \\
\hline$C(12)$ & $1156(7)$ & $1830(2)$ & $3433(7)$ & $46(2)$ \\
\hline$C(13)$ & $885(8)$ & $2034(2)$ & $4222(9)$ & $66(3)$ \\
\hline$C(14)$ & $320(8)$ & $1873(3)$ & $2403(9)$ & $64(3)$ \\
\hline$C(15)$ & $4958(6)$ & $2179(2)$ & $3177(6)$ & $34(2)$ \\
\hline$C(16)$ & $4294(7)$ & $2406(2)$ & $2578(7)$ & $38(2)$ \\
\hline$C(17)$ & $4762(8)$ & $2657(2)$ & $2251(7)$ & $44(2)$ \\
\hline$C(18)$ & $5863(8)$ & $2687(2)$ & $2499(8)$ & $48(2)$ \\
\hline$C(19)$ & $6506(7)$ & $2467(2)$ & $3098(7)$ & $44(2)$ \\
\hline$C(20)$ & $6091(6)$ & $2212(2)$ & $3451(6)$ & $34(2)$ \\
\hline$C(21)$ & $3069(7)$ & $2384(2)$ & $2310(8)$ & $53(3)$ \\
\hline$C(22)$ & $2652(8)$ & $2608(3)$ & $2927(11)$ & $89(5)$ \\
\hline$C(23)$ & $2532(9)$ & $2431(3)$ & $1176(9)$ & $89(4)$ \\
\hline$C(24)$ & $6830(7)$ & $1984(2)$ & $4171(7)$ & $43(2)$ \\
\hline$C(25)$ & $7464(9)$ & $2146(3)$ & $5151(8)$ & $69(3)$ \\
\hline$C(26)$ & $7584(7)$ & $1821(2)$ & $3678(8)$ & $54(3)$ \\
\hline$C(27)$ & $7017(7)$ & $1084(2)$ & $1964(7)$ & $39(2)$ \\
\hline$C(28)$ & $7096(6)$ & $936(2)$ & $2792(7)$ & $40(2)$ \\
\hline$C(30)$ & $5710(6)$ & $1593(2)$ & $42(7)$ & $39(2)$ \\
\hline$C(31)$ & $5493(7)$ & $1634(2)$ & $-1008(7)$ & $46(2)$ \\
\hline$C(32)$ & $5385(8)$ & $1384(2)$ & $-1663(8)$ & $55(3)$ \\
\hline$C(33)$ & $5451(9)$ & $1089(2)$ & $-1277(8)$ & $55(3)$ \\
\hline$C(34)$ & $5622(7)$ & $1036(2)$ & $-253(8)$ & $47(2)$ \\
\hline$C(35)$ & $5779(6)$ & $1291(2)$ & $418(6)$ & $34(2)$ \\
\hline$C(36)$ & $5893(7)$ & $1877(2)$ & $723(7)$ & $37(2)$ \\
\hline$C(37)$ & $5213(8)$ & $2155(2)$ & $248(7)$ & $50(2)$ \\
\hline$C(38)$ & $7089(7)$ & $1964(2)$ & $1025(8)$ & $53(3)$ \\
\hline C (39) & $5615(8)$ & $705(2)$ & $130(8)$ & $50(2)$ \\
\hline
\end{tabular}




\begin{tabular}{|c|c|c|c|c|}
\hline$C(40)$ & $4542(9)$ & $541(2)$ & $-379(10)$ & $74(3)$ \\
\hline$C(41)$ & $6534(9)$ & $512(2)$ & $-5(10)$ & $76(4)$ \\
\hline$C(42)$ & $6264(6)$ & $730(2)$ & $3988(7)$ & $34(2)$ \\
\hline$C(43)$ & $6091(7)$ & $415(2)$ & $3787(8)$ & $45(2)$ \\
\hline$C(44)$ & $6173(8)$ & $212(2)$ & $4561(8)$ & $55(3)$ \\
\hline$C(45)$ & $6407(8)$ & $311(2)$ & $5543(8)$ & $55(3)$ \\
\hline$C(46)$ & $6581(8)$ & $623(2)$ & $5774(8)$ & $54(3)$ \\
\hline$C(47)$ & $6534(7)$ & $841(2)$ & $4997(7)$ & $39(2)$ \\
\hline$C(48)$ & $5820(8)$ & $293(2)$ & $2704(8)$ & $51(3)$ \\
\hline$C(49)$ & $4719(10)$ & $136(4)$ & $2356(10)$ & $102(5)$ \\
\hline$C(50)$ & $6653(11)$ & $75(3)$ & $2546(11)$ & $95(4)$ \\
\hline$C(51)$ & $6810(7)$ & $1173(2)$ & $5264(7)$ & $44(2)$ \\
\hline$C(52)$ & $8036(8)$ & $1204(2)$ & $5710(8)$ & $59(3)$ \\
\hline$C(53)$ & $6255(8)$ & $1316(2)$ & $5978(8)$ & $56(3)$ \\
\hline$C(54)$ & $2521(7)$ & $1527(2)$ & $1463(7)$ & $46(2)$ \\
\hline$C(55)$ & $2317(7)$ & $1212(2)$ & $1611(7)$ & $42(2)$ \\
\hline$C(56)$ & $2846(7)$ & $1036(2)$ & $1086(8)$ & $49(2)$ \\
\hline$C(57)$ & $3332(7)$ & $1237(2)$ & $556(7)$ & $49(3)$ \\
\hline$C(58)$ & $3125(7)$ & $1536(2)$ & $771(7)$ & $44(2)$ \\
\hline$C(59)$ & $-761(12)$ & $1968(3)$ & $-2219(9)$ & $93(4)$ \\
\hline$C(60)$ & $-14(12)$ & $2024(3)$ & $-466(12)$ & $107(5)$ \\
\hline$C(61)$ & $-1575(10)$ & $1686(4)$ & $-1148(11)$ & $95(4)$ \\
\hline$C(62)$ & $-1453(10)$ & $1473(3)$ & $-306(12)$ & $89(4)$ \\
\hline$C(63)$ & $-1367(12)$ & $979(4)$ & $-1149(11)$ & $105(5)$ \\
\hline$C(64)$ & $-607(10)$ & $1038(3)$ & $593(11)$ & $90(4)$ \\
\hline$C(65)$ & $1504(18)$ & $535(5)$ & $-1286(18)$ & $165(8)$ \\
\hline$C(66)$ & $40(30)$ & $573(8)$ & $-2590(30)$ & $282(17)$ \\
\hline$C(67)$ & $1350(20)$ & $802(6)$ & $-2810(20)$ & $210(12)$ \\
\hline$C(68)$ & $1660(20)$ & $1089(7)$ & $-3110(20)$ & $208(11)$ \\
\hline$C(69)$ & $1022(19)$ & $1540(5)$ & $-3380(19)$ & $173(9)$ \\
\hline$C(70)$ & $2716(16)$ & $1488(4)$ & $-2259(16)$ & $148(7)$ \\
\hline$C(71)$ & $9318(19)$ & $35(6)$ & $5570(20)$ & 201 (11) \\
\hline$C(72)$ & $9217(16)$ & $184(4)$ & $4665(17)$ & $144(7)$ \\
\hline$C(73)$ & $9790(20)$ & $158(5)$ & $4000(15)$ & $174(9)$ \\
\hline$C(74)$ & $9650(30)$ & $308(9)$ & $2930(20)$ & $151(14)$ \\
\hline
\end{tabular}

Table 3. Bond lengths [A] and angles [deg] for cj309.

\begin{tabular}{ll}
\hline & \\
$\mathrm{Ga}(1)-\mathrm{N}(2)$ & $1.907(7)$ \\
$\mathrm{Ga}(1)-\mathrm{N}(1)$ & $1.910(7)$ \\
$\mathrm{Ga}(1)-\mathrm{Ga}(2)$ & $2.4549(13)$ \\
$\mathrm{Ga}(2)-\mathrm{N}(3)$ & $1.910(7)$ \\
$\mathrm{Ga}(2)-\mathrm{N}(4)$ & $1.912(7)$ \\
$\mathrm{K}(1)-\mathrm{N}(8)$ & $2.770(11)$ \\
$\mathrm{K}(1)-\mathrm{N}(6)$ & $2.787(9)$ \\
$\mathrm{K}(1)-\mathrm{N}(7)$ & $2.873(16)$ \\
$\mathrm{K}(1)-\mathrm{N}(5)$ & $2.930(9)$ \\
$\mathrm{K}(1)-\mathrm{C}(57)$ & $3.049(9)$ \\
$\mathrm{K}(1)-\mathrm{C}(58)$ & $3.108(9)$ \\
$\mathrm{K}(1)-\mathrm{C}(56)$ & $3.204(10)$ \\
$\mathrm{K}(1)-\mathrm{C}(55)$ & $3.323(9)$ \\
$\mathrm{K}(1)-\mathrm{C}(54)$ & $3.323(9)$ \\
$\mathrm{N}(1)-\mathrm{C}(1)$ & $1.402(10)$ \\
$\mathrm{N}(1)-\mathrm{C}(3)$ & $1.439(10)$ \\
$\mathrm{N}(2)-\mathrm{C}(2)$ & $1.391(11)$ \\
$\mathrm{N}(2)-\mathrm{C}(15)$ & $1.437(10)$ \\
$\mathrm{N}(3)-\mathrm{C}(27)$ & $1.430(11)$ \\
$\mathrm{N}(3)-\mathrm{C}(35)$ & $1.434(11)$
\end{tabular}




\begin{tabular}{|c|c|}
\hline$N(4)-C(28)$ & $1.398(10)$ \\
\hline$N(4)-C(42)$ & $1.424(10)$ \\
\hline$N(5)-C(60)$ & $1.435(16)$ \\
\hline$N(5)-C(59)$ & $1.451(14)$ \\
\hline$N(5)-C(61)$ & $1.493(16)$ \\
\hline$N(6)-C(64)$ & $1.431(16)$ \\
\hline$N(6)-C(62)$ & $1.444(15)$ \\
\hline$N(6)-C(63)$ & $1.450(16)$ \\
\hline$N(7)-C(67)$ & $1.30(3)$ \\
\hline$N(7)-C(65)$ & $1.41(2)$ \\
\hline$N(7)-C(66)$ & $1.52(4)$ \\
\hline$N(8)-C(69)$ & $1.31(2)$ \\
\hline$N(8)-C(68)$ & $1.44(3)$ \\
\hline$N(8)-C(70)$ & $1.47(2)$ \\
\hline$C(1)-C(2)$ & $1.348(11)$ \\
\hline$C(3)-C(4)$ & $1.415(12)$ \\
\hline$C(3)-C(8)$ & $1.429(12)$ \\
\hline$C(4)-C(5)$ & $1.384(12)$ \\
\hline$C(4)-C(9)$ & $1.534(13)$ \\
\hline$C(5)-C(6)$ & $1.398(14)$ \\
\hline$C(6)-C(7)$ & $1.347(14)$ \\
\hline$C(7)-C(8)$ & $1.412(12)$ \\
\hline$C(8)-C(12)$ & $1.492(13)$ \\
\hline$C(9)-C(11)$ & $1.504(13)$ \\
\hline$C(9)-C(10)$ & $1.523(15)$ \\
\hline$C(12)-C(14)$ & $1.522(14)$ \\
\hline$C(12)-C(13)$ & $1.524(13)$ \\
\hline$C(15)-C(16)$ & $1.402(12)$ \\
\hline$C(15)-C(20)$ & $1.421(11)$ \\
\hline$C(16)-C(17)$ & $1.386(12)$ \\
\hline$C(16)-C(21)$ & $1.533(12)$ \\
\hline$C(17)-C(18)$ & $1.381(13)$ \\
\hline$C(18)-C(19)$ & $1.370(13)$ \\
\hline$C(19)-C(20)$ & $1.380(12)$ \\
\hline$C(20)-C(24)$ & $1.522(12)$ \\
\hline$C(21)-C(22)$ & $1.496(15)$ \\
\hline$C(21)-C(23)$ & $1.527(15)$ \\
\hline$C(24)-C(25)$ & $1.527(13)$ \\
\hline$C(24)-C(26)$ & $1.530(12)$ \\
\hline$C(27)-C(28)$ & $1.290(12)$ \\
\hline$C(30)-C(35)$ & $1.396(11)$ \\
\hline$C(30)-C(31)$ & $1.404(13)$ \\
\hline$C(30)-C(36)$ & $1.522(12)$ \\
\hline$C(31)-C(32)$ & $1.386(13)$ \\
\hline$C(32)-C(33)$ & $1.377(14)$ \\
\hline$C(33)-C(34)$ & $1.384(14)$ \\
\hline$C(34)-C(35)$ & $1.414(12)$ \\
\hline$C(34)-C(39)$ & $1.528(13)$ \\
\hline$C(36)-C(37)$ & $1.520(12)$ \\
\hline$C(36)-C(38)$ & $1.536(12)$ \\
\hline$C(39)-C(41)$ & $1.521(14)$ \\
\hline$C(39)-C(40)$ & $1.537(14)$ \\
\hline$C(42)-C(43)$ & $1.394(12)$ \\
\hline$C(42)-C(47)$ & $1.415(12)$ \\
\hline$C(43)-C(44)$ & $1.363(13)$ \\
\hline$C(43)-C(48)$ & $1.524(14)$ \\
\hline$C(44)-C(45)$ & $1.367(14)$ \\
\hline$C(45)-C(46)$ & $1.388(13)$ \\
\hline$C(46)-C(47)$ & $1.414(13)$ \\
\hline$C(47)-C(51)$ & $1.499(12)$ \\
\hline$C(48)-C(50)$ & $1.507(15)$ \\
\hline
\end{tabular}




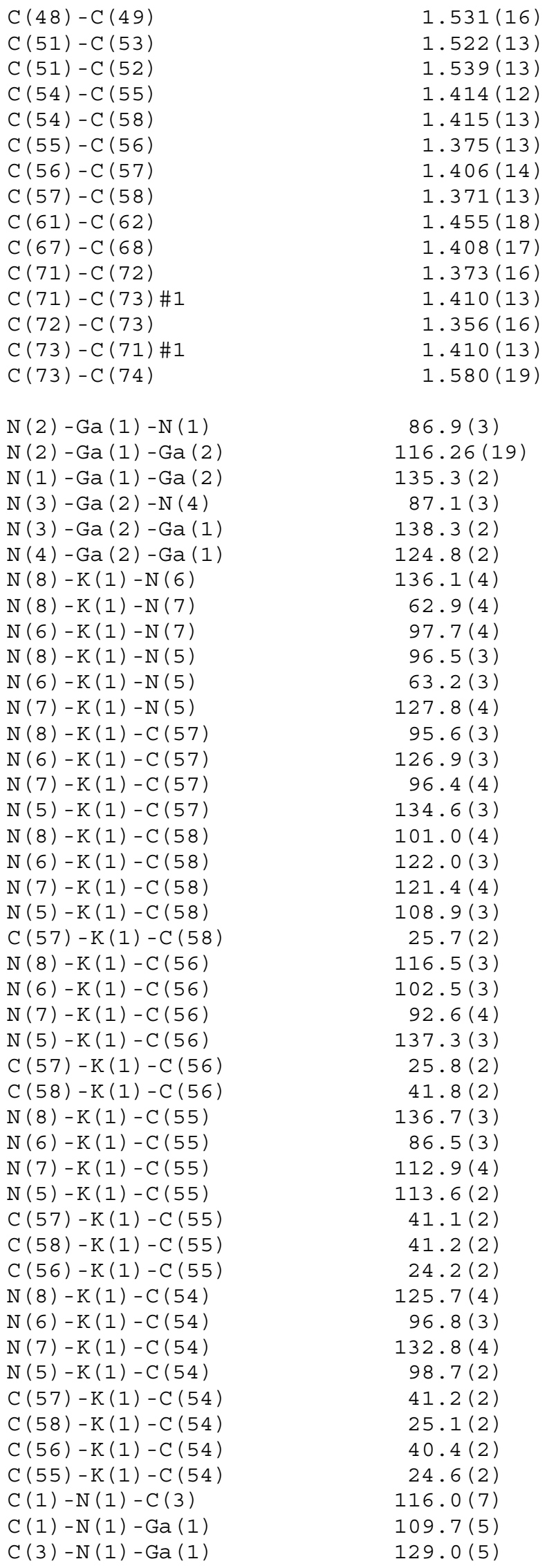

$1.531(16)$

$1.522(13)$

1.539 (13)

$1.414(12)$

1. $415(13)$

$1.375(13)$

$1.406(14)$

$1.371(13)$

$1.455(18)$

$1.408(17)$

$1.373(16)$

$1.410(13)$

$1.356(16)$

$1.410(13)$

1.580 (19)

$86.9(3)$

116.26 (19)

$135.3(2)$

$87.1(3)$

$138.3(2)$

$124.8(2)$

$136.1(4)$

$62.9(4)$

$97.7(4)$

$96.5(3)$

$63.2(3)$

$127.8(4)$

$95.6(3)$

$126.9(3)$

$96.4(4)$

$134.6(3)$

$101.0(4)$

$122.0(3)$

$121.4(4)$

$108.9(3)$

$25.7(2)$

$116.5(3)$

$102.5(3)$

$92.6(4)$

$137.3(3)$

$25.8(2)$

$41.8(2)$

$136.7(3)$

$86.5(3)$

$112.9(4)$

$113.6(2)$

$41.1(2)$

$41.2(2)$

$24.2(2)$

$125.7(4)$

$96.8(3)$

$132.8(4)$

$98.7(2)$

$41.2(2)$

25.1(2)

$40.4(2)$

$24.6(2)$

$116.0(7)$

$109.7(5)$

$129.0(5)$ 


\begin{tabular}{|c|c|}
\hline$C(2)-N(2)-C(15)$ & $116.5(7)$ \\
\hline $\mathrm{C}(2)-\mathrm{N}(2)-\mathrm{Ga}(1)$ & $108.9(5)$ \\
\hline $\mathrm{C}(15)-\mathrm{N}(2)-\mathrm{Ga}(1)$ & $133.4(6)$ \\
\hline$C(27)-N(3)-C(35)$ & $115.9(7)$ \\
\hline $\mathrm{C}(27)-\mathrm{N}(3)-\mathrm{Ga}(2)$ & $107.8(5)$ \\
\hline $\mathrm{C}(35)-\mathrm{N}(3)-\mathrm{Ga}(2)$ & $132.3(5)$ \\
\hline$C(28)-N(4)-C(42)$ & $117.2(7)$ \\
\hline $\mathrm{C}(28)-\mathrm{N}(4)-\mathrm{Ga}(2)$ & $108.1(5)$ \\
\hline $\mathrm{C}(42)-\mathrm{N}(4)-\mathrm{Ga}(2)$ & $133.3(5)$ \\
\hline$C(60)-N(5)-C(59)$ & $108.1(11)$ \\
\hline$C(60)-N(5)-C(61)$ & $113.8(11)$ \\
\hline$C(59)-N(5)-C(61)$ & $109.4(10)$ \\
\hline$C(60)-N(5)-K(1)$ & $99.9(7)$ \\
\hline $\mathrm{C}(59)-\mathrm{N}(5)-\mathrm{K}(1)$ & $116.9(7)$ \\
\hline $\mathrm{C}(61)-\mathrm{N}(5)-\mathrm{K}(1)$ & $108.7(7)$ \\
\hline$C(64)-N(6)-C(62)$ & $108.7(11)$ \\
\hline$C(64)-N(6)-C(63)$ & $107.7(11)$ \\
\hline$C(62)-N(6)-C(63)$ & $115.6(11)$ \\
\hline $\mathrm{C}(64)-\mathrm{N}(6)-\mathrm{K}(1)$ & $110.1(7)$ \\
\hline $\mathrm{C}(62)-\mathrm{N}(6)-\mathrm{K}(1)$ & $114.0(7)$ \\
\hline $\mathrm{C}(63)-\mathrm{N}(6)-\mathrm{K}(1)$ & $100.4(8)$ \\
\hline$C(67)-N(7)-C(65)$ & $120(2)$ \\
\hline$C(67)-N(7)-C(66)$ & $94(2)$ \\
\hline$C(65)-N(7)-C(66)$ & $97.2(19)$ \\
\hline $\mathrm{C}(67)-\mathrm{N}(7)-\mathrm{K}(1)$ & $114.6(16)$ \\
\hline $\mathrm{C}(65)-\mathrm{N}(7)-\mathrm{K}(1)$ & $103.8(13)$ \\
\hline $\mathrm{C}(66)-\mathrm{N}(7)-\mathrm{K}(1)$ & $127.0(16)$ \\
\hline$C(69)-N(8)-C(68)$ & $99.7(18)$ \\
\hline$C(69)-N(8)-C(70)$ & $111.2(16)$ \\
\hline$C(68)-N(8)-C(70)$ & $104.4(16)$ \\
\hline$C(69)-N(8)-K(1)$ & $122.2(13)$ \\
\hline $\mathrm{C}(68)-\mathrm{N}(8)-\mathrm{K}(1)$ & $113.9(13)$ \\
\hline $\mathrm{C}(70)-\mathrm{N}(8)-\mathrm{K}(1)$ & $104.3(11)$ \\
\hline $\mathrm{C}(2)-\mathrm{C}(1)-\mathrm{N}(1)$ & $116.0(8)$ \\
\hline $\mathrm{C}(1)-\mathrm{C}(2)-\mathrm{N}(2)$ & $118.4(8)$ \\
\hline$C(4)-C(3)-C(8)$ & $119.1(7)$ \\
\hline $\mathrm{C}(4)-\mathrm{C}(3)-\mathrm{N}(1)$ & $119.7(7)$ \\
\hline$C(8)-C(3)-N(1)$ & $121.3(8)$ \\
\hline$C(5)-C(4)-C(3)$ & $120.7(8)$ \\
\hline$C(5)-C(4)-C(9)$ & $119.0(8)$ \\
\hline$C(3)-C(4)-C(9)$ & $120.3(7)$ \\
\hline$C(4)-C(5)-C(6)$ & $119.5(9)$ \\
\hline$C(7)-C(6)-C(5)$ & $120.9(9)$ \\
\hline$C(6)-C(7)-C(8)$ & $122.1(9)$ \\
\hline$C(7)-C(8)-C(3)$ & $117.6(8)$ \\
\hline$C(7)-C(8)-C(12)$ & $118.9(8)$ \\
\hline$C(3)-C(8)-C(12)$ & $123.5(8)$ \\
\hline$C(11)-C(9)-C(10)$ & $109.4(8)$ \\
\hline$C(11)-C(9)-C(4)$ & $111.1(8)$ \\
\hline$C(10)-C(9)-C(4)$ & $110.3(8)$ \\
\hline$C(8)-C(12)-C(14)$ & $111.9(8)$ \\
\hline$C(8)-C(12)-C(13)$ & $112.8(9)$ \\
\hline$C(14)-C(12)-C(13)$ & $110.1(8)$ \\
\hline$C(16)-C(15)-C(20)$ & $119.8(7)$ \\
\hline$C(16)-C(15)-N(2)$ & $119.9(7)$ \\
\hline$C(20)-C(15)-N(2)$ & $120.3(7)$ \\
\hline$C(17)-C(16)-C(15)$ & $118.9(8)$ \\
\hline$C(17)-C(16)-C(21)$ & $120.1(8)$ \\
\hline$C(15)-C(16)-C(21)$ & $121.0(8)$ \\
\hline$C(18)-C(17)-C(16)$ & $121.6(8)$ \\
\hline$C(19)-C(18)-C(17)$ & $119.1(8)$ \\
\hline
\end{tabular}




\begin{tabular}{|c|c|}
\hline$C(18)-C(19)-C(20)$ & $122.2(8)$ \\
\hline$C(19)-C(20)-C(15)$ & $118.4(8)$ \\
\hline$C(19)-C(20)-C(24)$ & $120.6(8)$ \\
\hline$C(15)-C(20)-C(24)$ & $120.9(7)$ \\
\hline$C(22)-C(21)-C(23)$ & $111.5(10)$ \\
\hline$C(22)-C(21)-C(16)$ & $110.9(8)$ \\
\hline$C(23)-C(21)-C(16)$ & $111.2(9)$ \\
\hline$C(20)-C(24)-C(25)$ & $110.5(7)$ \\
\hline$C(20)-C(24)-C(26)$ & $111.9(8)$ \\
\hline$C(25)-C(24)-C(26)$ & $110.9(8)$ \\
\hline $\mathrm{C}(28)-\mathrm{C}(27)-\mathrm{N}(3)$ & $117.5(8)$ \\
\hline $\mathrm{C}(27)-\mathrm{C}(28)-\mathrm{N}(4)$ & $119.2(8)$ \\
\hline$C(35)-C(30)-C(31)$ & $118.2(8)$ \\
\hline$C(35)-C(30)-C(36)$ & $122.8(8)$ \\
\hline$C(31)-C(30)-C(36)$ & $119.0(8)$ \\
\hline$C(32)-C(31)-C(30)$ & $121.9(9)$ \\
\hline$C(33)-C(32)-C(31)$ & $119.0(10)$ \\
\hline$C(32)-C(33)-C(34)$ & $121.3(9)$ \\
\hline$C(33)-C(34)-C(35)$ & $119.5(9)$ \\
\hline$C(33)-C(34)-C(39)$ & $119.4(8)$ \\
\hline$C(35)-C(34)-C(39)$ & $121.1(9)$ \\
\hline$C(30)-C(35)-C(34)$ & $120.1(8)$ \\
\hline $\mathrm{C}(30)-\mathrm{C}(35)-\mathrm{N}(3)$ & $119.6(7)$ \\
\hline$C(34)-C(35)-N(3)$ & $120.3(8)$ \\
\hline$C(37)-C(36)-C(30)$ & $114.1(8)$ \\
\hline$C(37)-C(36)-C(38)$ & $109.8(7)$ \\
\hline$C(30)-C(36)-C(38)$ & $108.6(7)$ \\
\hline$C(41)-C(39)-C(34)$ & $112.5(8)$ \\
\hline$C(41)-C(39)-C(40)$ & $109.7(8)$ \\
\hline$C(34)-C(39)-C(40)$ & $111.9(9)$ \\
\hline$C(43)-C(42)-C(47)$ & $120.2(8)$ \\
\hline$C(43)-C(42)-N(4)$ & $121.4(8)$ \\
\hline $\mathrm{C}(47)-\mathrm{C}(42)-\mathrm{N}(4)$ & $118.2(7)$ \\
\hline$C(44)-C(43)-C(42)$ & $120.1(9)$ \\
\hline$C(44)-C(43)-C(48)$ & $119.4(8)$ \\
\hline$C(42)-C(43)-C(48)$ & $120.6(8)$ \\
\hline$C(43)-C(44)-C(45)$ & $121.4(9)$ \\
\hline$C(44)-C(45)-C(46)$ & $120.3(9)$ \\
\hline$C(45)-C(46)-C(47)$ & $120.3(10)$ \\
\hline$C(46)-C(47)-C(42)$ & $117.7(8)$ \\
\hline$C(46)-C(47)-C(51)$ & $119.9(8)$ \\
\hline$C(42)-C(47)-C(51)$ & $122.3(7)$ \\
\hline$C(50)-C(48)-C(43)$ & $113.3(9)$ \\
\hline$C(50)-C(48)-C(49)$ & $109.1(10)$ \\
\hline$C(43)-C(48)-C(49)$ & $112.3(8)$ \\
\hline$C(47)-C(51)-C(53)$ & $114.8(8)$ \\
\hline$C(47)-C(51)-C(52)$ & $109.0(8)$ \\
\hline$C(53)-C(51)-C(52)$ & $110.4(8)$ \\
\hline$C(55)-C(54)-C(58)$ & $106.9(8)$ \\
\hline$C(55)-C(54)-K(1)$ & $77.7(5)$ \\
\hline$C(58)-C(54)-K(1)$ & $68.9(5)$ \\
\hline$C(56)-C(55)-C(54)$ & $108.1(8)$ \\
\hline$C(56)-C(55)-K(1)$ & $73.1(5)$ \\
\hline$C(54)-C(55)-K(1)$ & $77.7(5)$ \\
\hline$C(55)-C(56)-C(57)$ & $108.2(8)$ \\
\hline$C(55)-C(56)-K(1)$ & $82.7(5)$ \\
\hline$C(57)-C(56)-K(1)$ & $70.9(5)$ \\
\hline$C(58)-C(57)-C(56)$ & $108.6(9)$ \\
\hline$C(58)-C(57)-K(1)$ & $79.5(5)$ \\
\hline$C(56)-C(57)-K(1)$ & $83.3(5)$ \\
\hline$C(57)-C(58)-C(54)$ & $108.1(8)$ \\
\hline
\end{tabular}




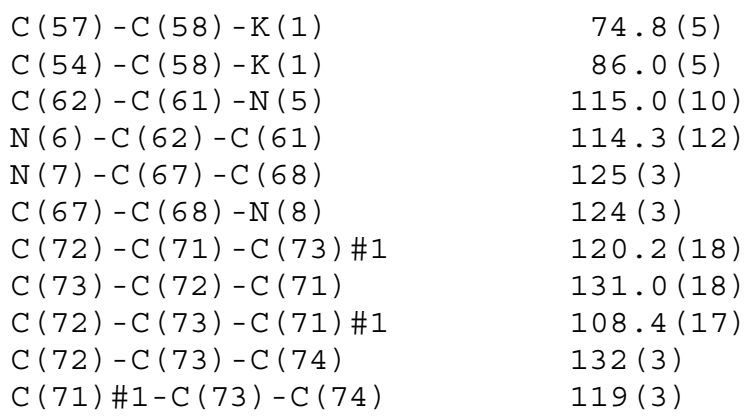

Symmetry transformations used to generate equivalent atoms: $\# 1-x+2,-y,-z+1$

Table 4. Anisotropic displacement parameters (A^2 x 10^3) for cj309. The anisotropic displacement factor exponent takes the form:

$-2 \mathrm{pi}^{\wedge} 2\left[\mathrm{~h}^{\wedge} 2 \mathrm{a}^{\star \wedge} 2 \mathrm{U} 11+\ldots+2 \mathrm{~h} \mathrm{k} a * \mathrm{~b} * \mathrm{U} 12\right]$

\begin{tabular}{|c|c|c|c|c|c|c|}
\hline & U11 & $\mathrm{U} 22$ & U33 & $\mathrm{U} 23$ & U13 & U12 \\
\hline $\mathrm{Ga}(1)$ & $27(1)$ & $33(1)$ & $34(1)$ & $-2(1)$ & $10(1)$ & $2(1)$ \\
\hline $\mathrm{Ga}(2)$ & $29(1)$ & $33(1)$ & $32(1)$ & $-1(1)$ & $10(1)$ & $3(1)$ \\
\hline $\mathrm{K}(1)$ & $36(1)$ & $79(2)$ & $40(1)$ & $0(1)$ & $11(1)$ & $8(1)$ \\
\hline $\mathrm{N}(1)$ & $31(4)$ & $39(4)$ & $29(4)$ & $-5(3)$ & $8(3)$ & $-3(3)$ \\
\hline $\mathrm{N}(2)$ & $34(4)$ & $36(4)$ & $37(5)$ & $1(3)$ & $16(3)$ & $2(3)$ \\
\hline $\mathrm{N}(3)$ & $44(4)$ & $38(4)$ & $28(4)$ & $3(3)$ & $14(3)$ & $3(3)$ \\
\hline $\mathrm{N}(4)$ & $32(4)$ & $36(4)$ & $36(5)$ & $1(3)$ & $13(3)$ & $3(3)$ \\
\hline $\mathrm{N}(5)$ & $65(6)$ & $74(6)$ & $45(6)$ & $-3(4)$ & $14(5)$ & $13(5)$ \\
\hline $\mathrm{N}(6)$ & $50(5)$ & $82(7)$ & $94(9)$ & $-4(6)$ & $38(6)$ & $-3(5)$ \\
\hline $\mathrm{N}(7)$ & $120(11)$ & $159(13)$ & $146(14)$ & $-5(11)$ & $82(11)$ & $0(10)$ \\
\hline $\mathrm{N}(8)$ & $102(9)$ & $153(11)$ & $74(8)$ & $27(8)$ & $62(7)$ & $31(8)$ \\
\hline$C(1)$ & $36(5)$ & $45(5)$ & $26(5)$ & $-4(4)$ & $13(4)$ & $-2(4)$ \\
\hline$C(2)$ & $30(4)$ & $43(5)$ & $26(5)$ & $1(4)$ & $-1(4)$ & $2(4)$ \\
\hline$C(3)$ & $22(4)$ & $53(5)$ & $41(6)$ & $-14(4)$ & $19(4)$ & $-7(4)$ \\
\hline$C(4)$ & $53(6)$ & $37(5)$ & $49(6)$ & $-10(4)$ & $28(5)$ & $-5(4)$ \\
\hline$C(5)$ & $54(6)$ & $42(5)$ & $85(9)$ & $-7(5)$ & $38(6)$ & $-14(5)$ \\
\hline$C(6)$ & $41(6)$ & $68(7)$ & $80(9)$ & $-17(6)$ & $25(6)$ & $-21(5)$ \\
\hline$C(7)$ & $33(5)$ & $60(7)$ & $61(7)$ & $-18(5)$ & $16(5)$ & $-3(4)$ \\
\hline$C(8)$ & $33(5)$ & $49(5)$ & $47(6)$ & $-17(4)$ & $14(4)$ & $-3(4)$ \\
\hline$C(9)$ & $41(5)$ & $40(5)$ & $60(7)$ & $5(4)$ & $23(5)$ & $2(4)$ \\
\hline$C(10)$ & $62(7)$ & $88(8)$ & $67(9)$ & $-5(6)$ & $14(6)$ & $10(6)$ \\
\hline$C(11)$ & $74(7)$ & $58(7)$ & $73(8)$ & $-2(6)$ & $32(7)$ & $12(6)$ \\
\hline$C(12)$ & $33(5)$ & $49(6)$ & $49(6)$ & $-4(4)$ & $3(5)$ & $0(4)$ \\
\hline$C(13)$ & $44(6)$ & $61(7)$ & $88(9)$ & $-18(6)$ & $14(6)$ & $9(5)$ \\
\hline$C(14)$ & $41(6)$ & $78(7)$ & $65(8)$ & $-2(6)$ & $6(6)$ & $11(5)$ \\
\hline$C(15)$ & $38(5)$ & $37(5)$ & $26(5)$ & $-8(4)$ & $9(4)$ & $-1(4)$ \\
\hline$C(16)$ & $45(5)$ & $37(5)$ & $28(5)$ & $1(4)$ & $5(4)$ & $9(4)$ \\
\hline$C(17)$ & $55(6)$ & $38(5)$ & $35(6)$ & $1(4)$ & $8(5)$ & $4(4)$ \\
\hline$C(18)$ & $57(6)$ & $33(5)$ & $53(7)$ & $0(4)$ & $17(5)$ & $-7(4)$ \\
\hline$C(19)$ & $44(5)$ & $44(5)$ & $49(6)$ & $-7(5)$ & $19(5)$ & $-11(4)$ \\
\hline$C(20)$ & $35(5)$ & $39(5)$ & $28(5)$ & $-2(4)$ & $11(4)$ & $2(4)$ \\
\hline$C(21)$ & $36(5)$ & $43(5)$ & $72(8)$ & $3(5)$ & $6(5)$ & $3(4)$ \\
\hline$C(22)$ & $36(6)$ & $85(8)$ & $134(13)$ & $-42(8)$ & $6(7)$ & $13(6)$ \\
\hline$C(23)$ & $61(8)$ & $111(10)$ & $68(10)$ & $22(7)$ & $-23(7)$ & $-10(7)$ \\
\hline$C(24)$ & $38(5)$ & $46(5)$ & $42(6)$ & $-5(4)$ & $7(5)$ & $-2(4)$ \\
\hline
\end{tabular}




\begin{tabular}{|c|c|c|c|c|c|c|}
\hline$C(25)$ & $72(7)$ & $72(7)$ & $50(8)$ & $-3(6)$ & $1(6)$ & $13(6)$ \\
\hline$C(26)$ & $40(5)$ & $59(6)$ & $62(7)$ & $0(5)$ & $13(5)$ & $8(5)$ \\
\hline$C(27)$ & $39(5)$ & $38(5)$ & $41(6)$ & $11(4)$ & $13(4)$ & $10(4)$ \\
\hline$C(28)$ & $29(4)$ & $37(5)$ & $56(7)$ & $0(4)$ & $14(5)$ & $6(4)$ \\
\hline$C(30)$ & $31(5)$ & $40(5)$ & $44(6)$ & $-2(4)$ & $10(4)$ & $-2(4)$ \\
\hline$C(31)$ & $52(6)$ & $53(6)$ & $33(6)$ & $8(4)$ & $12(5)$ & $6(4)$ \\
\hline$C(32)$ & $61(6)$ & $76(7)$ & $33(6)$ & $-4(5)$ & $21(5)$ & $1(5)$ \\
\hline$C(33)$ & $77(7)$ & $53(6)$ & $41(7)$ & $-6(5)$ & $27(6)$ & $-3(5)$ \\
\hline$C(34)$ & $44(5)$ & $47(5)$ & $56(7)$ & $-10(5)$ & $25(5)$ & $-3(4)$ \\
\hline$C(35)$ & $34(5)$ & $41(5)$ & $31(5)$ & $1(4)$ & $15(4)$ & $2(4)$ \\
\hline$C(36)$ & $44(5)$ & $45(5)$ & $30(5)$ & $4(4)$ & $24(4)$ & $7(4)$ \\
\hline$C(37)$ & $69(7)$ & $40(5)$ & $40(6)$ & $5(4)$ & $17(5)$ & $11(5)$ \\
\hline$C(38)$ & $48(6)$ & $60(6)$ & $55(7)$ & $-7(5)$ & $21(5)$ & $-3(5)$ \\
\hline$C(39)$ & $62(6)$ & $46(5)$ & $46(6)$ & $-10(4)$ & $22(5)$ & $-11(5)$ \\
\hline$C(40)$ & $72(8)$ & $59(7)$ & $95(10)$ & $-19(6)$ & $33(7)$ & $-10(6)$ \\
\hline$C(41)$ & $73(8)$ & $50(6)$ & $104(11)$ & $-8(6)$ & $26(7)$ & $1(6)$ \\
\hline$C(42)$ & $20(4)$ & $39(5)$ & $38(6)$ & $7(4)$ & $2(4)$ & $6(3)$ \\
\hline$C(43)$ & $37(5)$ & $36(5)$ & $59(7)$ & $4(5)$ & $13(5)$ & $0(4)$ \\
\hline$C(44)$ & $69(7)$ & $35(5)$ & $60(8)$ & $3(5)$ & $18(6)$ & $5(5)$ \\
\hline$C(45)$ & $63(7)$ & $46(6)$ & $60(8)$ & $21(5)$ & $24(6)$ & $13(5)$ \\
\hline$C(46)$ & $57(6)$ & $66(7)$ & $36(6)$ & $9(5)$ & $8(5)$ & $7(5)$ \\
\hline$C(47)$ & $41(5)$ & $49(5)$ & $25(5)$ & $10(4)$ & $6(4)$ & $7(4)$ \\
\hline$C(48)$ & $62(6)$ & $41(5)$ & $58(7)$ & $-8(5)$ & $27(6)$ & $1(5)$ \\
\hline$C(49)$ & $80(9)$ & $173(15)$ & $60(9)$ & $-44(9)$ & $32(8)$ & $-14(9)$ \\
\hline$C(50)$ & $104(10)$ & $108(10)$ & $73(10)$ & $-33(8)$ & $24(8)$ & $22(8)$ \\
\hline$C(51)$ & $54(6)$ & $48(5)$ & $29(5)$ & $2(4)$ & $9(5)$ & $-2(4)$ \\
\hline$C(52)$ & $46(6)$ & $74(7)$ & $53(7)$ & $-3(5)$ & $8(5)$ & $-4(5)$ \\
\hline$C(53)$ & $50(6)$ & $64(6)$ & $53(7)$ & $-14(5)$ & $13(5)$ & $-1(5)$ \\
\hline$C(54)$ & $31(5)$ & $53(6)$ & $45(6)$ & $-12(4)$ & $-2(5)$ & $7(4)$ \\
\hline$C(55)$ & $29(5)$ & $65(6)$ & $26(5)$ & $-3(4)$ & $0(4)$ & $-9(4)$ \\
\hline$C(56)$ & $43(5)$ & $46(5)$ & $50(7)$ & $-2(5)$ & $3(5)$ & $-1(4)$ \\
\hline$C(57)$ & $33(5)$ & $77(7)$ & $31(6)$ & $-19(5)$ & $0(4)$ & $1(5)$ \\
\hline$C(58)$ & $37(5)$ & $56(6)$ & $30(6)$ & $7(4)$ & $-3(4)$ & $-12(4)$ \\
\hline$C(59)$ & $120(11)$ & $115(11)$ & $35(8)$ & $9(7)$ & $9(8)$ & $18(9)$ \\
\hline$C(60)$ & $109(6)$ & $105(6)$ & $101(6)$ & $-4(5)$ & $25(5)$ & $2(4)$ \\
\hline$C(61)$ & $59(8)$ & $148(13)$ & $72(10)$ & $7(9)$ & $13(7)$ & $41(8)$ \\
\hline$C(62)$ & $58(7)$ & $116(11)$ & $107(12)$ & $-9(9)$ & $46(8)$ & $6(7)$ \\
\hline$C(63)$ & $99(11)$ & $136(13)$ & $76(11)$ & $-14(9)$ & $18(9)$ & $-15(10)$ \\
\hline$C(64)$ & $67(8)$ & $116(11)$ & $91(11)$ & $-3(9)$ & $30(8)$ & $-21(7)$ \\
\hline$C(65)$ & $164(9)$ & $163(9)$ & $166(9)$ & $-5(5)$ & $47(5)$ & $2(5)$ \\
\hline$C(66)$ & $282(18)$ & $281(18)$ & $282(18)$ & $-1(5)$ & $85(7)$ & $4(5)$ \\
\hline$C(67)$ & $212(12)$ & $209(12)$ & $211(12)$ & $-5(5)$ & $69(6)$ & $1(5)$ \\
\hline$C(68)$ & $211(12)$ & $209(12)$ & $205(12)$ & $-1(5)$ & $68(6)$ & $-1(5)$ \\
\hline$C(69)$ & $171(10)$ & $178(10)$ & $172(10)$ & $-1(5)$ & $54(5)$ & $3(5)$ \\
\hline$C(70)$ & $144(8)$ & $150(8)$ & $148(8)$ & $6(5)$ & $44(5)$ & $-3(5)$ \\
\hline
\end{tabular}

Table 5. Hydrogen coordinates $\left(x 0^{\wedge} 4\right)$ and isotropic displacement parameters $\left(A^{\wedge} 2 \times 10^{\wedge} 3\right)$ for $\mathrm{cj}^{3} 309$.

\begin{tabular}{|c|c|c|c|c|}
\hline & $\mathrm{x}$ & $\mathrm{Y}$ & z & $\mathrm{U}(\mathrm{eq})$ \\
\hline $\mathrm{H}(1)$ & 3296 & 1787 & 5231 & 42 \\
\hline $\mathrm{H}(2)$ & 4425 & 2150 & 4819 & 42 \\
\hline $\mathrm{H}(5)$ & 1466 & 655 & 4470 & 68 \\
\hline $\mathrm{H}(6)$ & -194 & 911 & 3962 & 74 \\
\hline $\mathrm{H}(7)$ & -290 & 1427 & 3611 & 61 \\
\hline
\end{tabular}




\begin{tabular}{|c|c|c|c|c|}
\hline $\mathrm{H}(9)$ & 3992 & 992 & 4488 & 55 \\
\hline $\mathrm{H}(10 \mathrm{~A})$ & 3181 & 639 & 5850 & 111 \\
\hline $\mathrm{H}(10 \mathrm{~B})$ & 3812 & 960 & 6127 & 111 \\
\hline $\mathrm{H}(10 \mathrm{C})$ & 4423 & 658 & 5918 & 111 \\
\hline $\mathrm{H}(11 \mathrm{~A})$ & 2799 & 429 & 4066 & 99 \\
\hline $\mathrm{H}(11 \mathrm{~B})$ & 4062 & 452 & 4229 & 99 \\
\hline $\mathrm{H}(11 \mathrm{C})$ & 3232 & 617 & 3272 & 99 \\
\hline $\mathrm{H}(12)$ & 1857 & 1904 & 3370 & 55 \\
\hline $\mathrm{H}(13 \mathrm{~A})$ & 164 & 1983 & 4248 & 99 \\
\hline $\mathrm{H}(13 \mathrm{~B})$ & 910 & 2252 & 4036 & 99 \\
\hline $\mathrm{H}(13 \mathrm{C})$ & 1408 & 1997 & 4888 & 99 \\
\hline $\mathrm{H}(14 \mathrm{~A})$ & 486 & 1735 & 1907 & 96 \\
\hline $\mathrm{H}(14 \mathrm{~B})$ & 329 & 2088 & 2184 & 96 \\
\hline $\mathrm{H}(14 \mathrm{C})$ & -393 & 1823 & 2455 & 96 \\
\hline $\mathrm{H}(17)$ & 4315 & 2811 & 1847 & 53 \\
\hline $\mathrm{H}(18)$ & 6169 & 2859 & 2258 & 57 \\
\hline $\mathrm{H}(19)$ & 7263 & 2490 & 3275 & 53 \\
\hline $\mathrm{H}(21)$ & 2889 & 2171 & 2486 & 63 \\
\hline $\mathrm{H}(22 \mathrm{~A})$ & 2981 & 2564 & 3649 & 134 \\
\hline $\mathrm{H}(22 \mathrm{~B})$ & 1871 & 2586 & 2754 & 134 \\
\hline $\mathrm{H}(22 \mathrm{C})$ & 2830 & 2819 & 2780 & 134 \\
\hline $\mathrm{H}(23 \mathrm{~A})$ & 2681 & 2641 & 985 & 134 \\
\hline $\mathrm{H}(23 \mathrm{~B})$ & 1755 & 2403 & 1019 & 134 \\
\hline $\mathrm{H}(23 \mathrm{C})$ & 2815 & 2280 & 795 & 134 \\
\hline $\mathrm{H}(24)$ & 6371 & 1822 & 4350 & 52 \\
\hline $\mathrm{H}(25 \mathrm{~A})$ & 7912 & 2309 & 4995 & 103 \\
\hline $\mathrm{H}(25 \mathrm{~B})$ & 7922 & 1995 & 5613 & 103 \\
\hline $\mathrm{H}(25 \mathrm{C})$ & 6964 & 2238 & 5473 & 103 \\
\hline $\mathrm{H}(26 \mathrm{~A})$ & 7160 & 1711 & 3068 & 81 \\
\hline $\mathrm{H}(26 \mathrm{~B})$ & 8034 & 1673 & 4158 & 81 \\
\hline $\mathrm{H}(26 \mathrm{C})$ & 8042 & 1975 & 3491 & 81 \\
\hline $\mathrm{H}(27)$ & 7594 & 1089 & 1680 & 47 \\
\hline $\mathrm{H}(28)$ & 7726 & 821 & 3118 & 48 \\
\hline $\mathrm{H}(31)$ & 5419 & 1838 & -1276 & 56 \\
\hline $\mathrm{H}(32)$ & 5267 & 1417 & -2367 & 66 \\
\hline $\mathrm{H}(33)$ & 5377 & 917 & -1723 & 66 \\
\hline $\mathrm{H}(36)$ & 5714 & 1821 & 1354 & 44 \\
\hline $\mathrm{H}(37 \mathrm{~A})$ & 4454 & 2095 & 19 & 75 \\
\hline $\mathrm{H}(37 \mathrm{~B})$ & 5427 & 2228 & -333 & 75 \\
\hline $\mathrm{H}(37 \mathrm{C})$ & 5318 & 2322 & 751 & 75 \\
\hline $\mathrm{H}(38 \mathrm{~A})$ & 7523 & 1783 & 1328 & 79 \\
\hline $\mathrm{H}(38 \mathrm{~B})$ & 7231 & 2133 & 1518 & 79 \\
\hline $\mathrm{H}(38 \mathrm{C})$ & 7274 & 2029 & 421 & 79 \\
\hline $\mathrm{H}(39)$ & 5706 & 716 & 873 & 60 \\
\hline $\mathrm{H}(40 \mathrm{~A})$ & 4450 & 515 & -1104 & 111 \\
\hline $\mathrm{H}(40 \mathrm{~B})$ & 3952 & 666 & -291 & 111 \\
\hline $\mathrm{H}(40 \mathrm{C})$ & 4541 & 338 & -65 & 111 \\
\hline $\mathrm{H}(41 \mathrm{~A})$ & 6478 & 501 & -727 & 114 \\
\hline $\mathrm{H}(41 \mathrm{~B})$ & 6498 & 302 & 254 & 114 \\
\hline $\mathrm{H}(41 \mathrm{C})$ & 7220 & 607 & 370 & 114 \\
\hline $\mathrm{H}(44)$ & 6064 & -3 & 4416 & 66 \\
\hline $\mathrm{H}(45)$ & 6452 & 166 & 6069 & 67 \\
\hline $\mathrm{H}(46)$ & 6731 & 691 & 6456 & 65 \\
\hline $\mathrm{H}(48)$ & 5788 & 476 & 2252 & 62 \\
\hline $\mathrm{H}(49 \mathrm{~A})$ & 4717 & -43 & 2794 & 153 \\
\hline $\mathrm{H}(49 \mathrm{~B})$ & 4572 & 65 & 1654 & 153 \\
\hline $\mathrm{H}(49 \mathrm{C})$ & 4165 & 283 & 2398 & 153 \\
\hline $\mathrm{H}(50 \mathrm{~A})$ & 6609 & -124 & 2875 & 143 \\
\hline $\mathrm{H}(50 \mathrm{~B})$ & 7369 & 164 & 2843 & 143 \\
\hline $\mathrm{H}(50 \mathrm{C})$ & 6524 & 42 & 1818 & 143 \\
\hline $\mathrm{H}(51)$ & 6587 & 1295 & 4618 & 53 \\
\hline
\end{tabular}




\begin{tabular}{|c|c|c|c|c|}
\hline $\mathrm{H}(52 \mathrm{~A})$ & 8276 & 1098 & 6366 & 89 \\
\hline $\mathrm{H}(52 \mathrm{~B})$ & 8230 & 1423 & 5801 & 89 \\
\hline $\mathrm{H}(52 \mathrm{C})$ & 8383 & 1110 & 5245 & 89 \\
\hline $\mathrm{H}(53 \mathrm{~A})$ & 5476 & 1314 & 5653 & 84 \\
\hline $\mathrm{H}(53 \mathrm{~B})$ & 6502 & 1529 & 6133 & 84 \\
\hline $\mathrm{H}(53 \mathrm{C})$ & 6429 & 1195 & 6607 & 84 \\
\hline $\mathrm{H}(54)$ & 2296 & 1701 & 1771 & 55 \\
\hline $\mathrm{H}(55)$ & 1890 & 1136 & 2005 & 50 \\
\hline $\mathrm{H}(56)$ & 2877 & 816 & 1082 & 59 \\
\hline $\mathrm{H}(57)$ & 3736 & 1175 & 122 & 59 \\
\hline $\mathrm{H}(58)$ & 3348 & 1717 & 501 & 53 \\
\hline $\mathrm{H}(59 \mathrm{~A})$ & -1156 & 2160 & -2204 & 140 \\
\hline $\mathrm{H}(59 \mathrm{~B})$ & -1191 & 1834 & -2762 & 140 \\
\hline $\mathrm{H}(59 \mathrm{C})$ & -80 & 2017 & -2344 & 140 \\
\hline $\mathrm{H}(60 \mathrm{~A})$ & 663 & 2090 & -566 & 160 \\
\hline $\mathrm{H}(60 \mathrm{~B})$ & 133 & 1922 & 195 & 160 \\
\hline $\mathrm{H}(60 \mathrm{C})$ & -474 & 2205 & -490 & 160 \\
\hline $\mathrm{H}(61 \mathrm{~A})$ & -2025 & 1862 & -1066 & 114 \\
\hline $\mathrm{H}(61 \mathrm{~B})$ & -1964 & 1578 & -1785 & 114 \\
\hline $\mathrm{H}(62 \mathrm{~A})$ & -1093 & 1583 & 335 & 106 \\
\hline $\mathrm{H}(62 \mathrm{~B})$ & -2174 & 1411 & -286 & 106 \\
\hline $\mathrm{H}(63 \mathrm{~A})$ & -935 & 790 & -1070 & 158 \\
\hline $\mathrm{H}(63 \mathrm{~B})$ & -1440 & 1072 & -1813 & 158 \\
\hline $\mathrm{H}(63 \mathrm{C})$ & -2080 & 928 & -1099 & 158 \\
\hline $\mathrm{H}(64 \mathrm{~A})$ & -209 & 1177 & 1140 & 135 \\
\hline $\mathrm{H}(64 \mathrm{~B})$ & -171 & 855 & 577 & 135 \\
\hline $\mathrm{H}(64 \mathrm{C})$ & -1278 & 975 & 713 & 135 \\
\hline $\mathrm{H}(65 \mathrm{~A})$ & 1810 & 371 & -1604 & 247 \\
\hline $\mathrm{H}(65 \mathrm{~B})$ & 963 & 446 & -1008 & 247 \\
\hline $\mathrm{H}(65 \mathrm{C})$ & 2075 & 631 & -738 & 247 \\
\hline $\mathrm{H}(66 \mathrm{~A})$ & 275 & 366 & -2731 & 423 \\
\hline $\mathrm{H}(66 \mathrm{~B})$ & -325 & 676 & -3232 & 423 \\
\hline $\mathrm{H}(66 \mathrm{C})$ & -444 & 554 & -2179 & 423 \\
\hline $\mathrm{H}(67 \mathrm{~A})$ & 774 & 721 & -3394 & 252 \\
\hline $\mathrm{H}(67 \mathrm{~B})$ & 1971 & 662 & -2713 & 252 \\
\hline $\mathrm{H}(68 \mathrm{~A})$ & 1228 & 1117 & -3830 & 249 \\
\hline $\mathrm{H}(68 \mathrm{~B})$ & 2414 & 1065 & -3106 & 249 \\
\hline $\mathrm{H}(69 \mathrm{~A})$ & 890 & 1746 & -3146 & 260 \\
\hline $\mathrm{H}(69 \mathrm{~B})$ & 335 & 1436 & -3688 & 260 \\
\hline $\mathrm{H}(69 \mathrm{C})$ & 1407 & 1561 & -3883 & 260 \\
\hline $\mathrm{H}(70 \mathrm{~A})$ & 2974 & 1521 & -2846 & 221 \\
\hline $\mathrm{H}(70 \mathrm{~B})$ & 3168 & 1334 & -1807 & 221 \\
\hline $\mathrm{H}(70 \mathrm{C})$ & 2748 & 1684 & -1892 & 221 \\
\hline
\end{tabular}




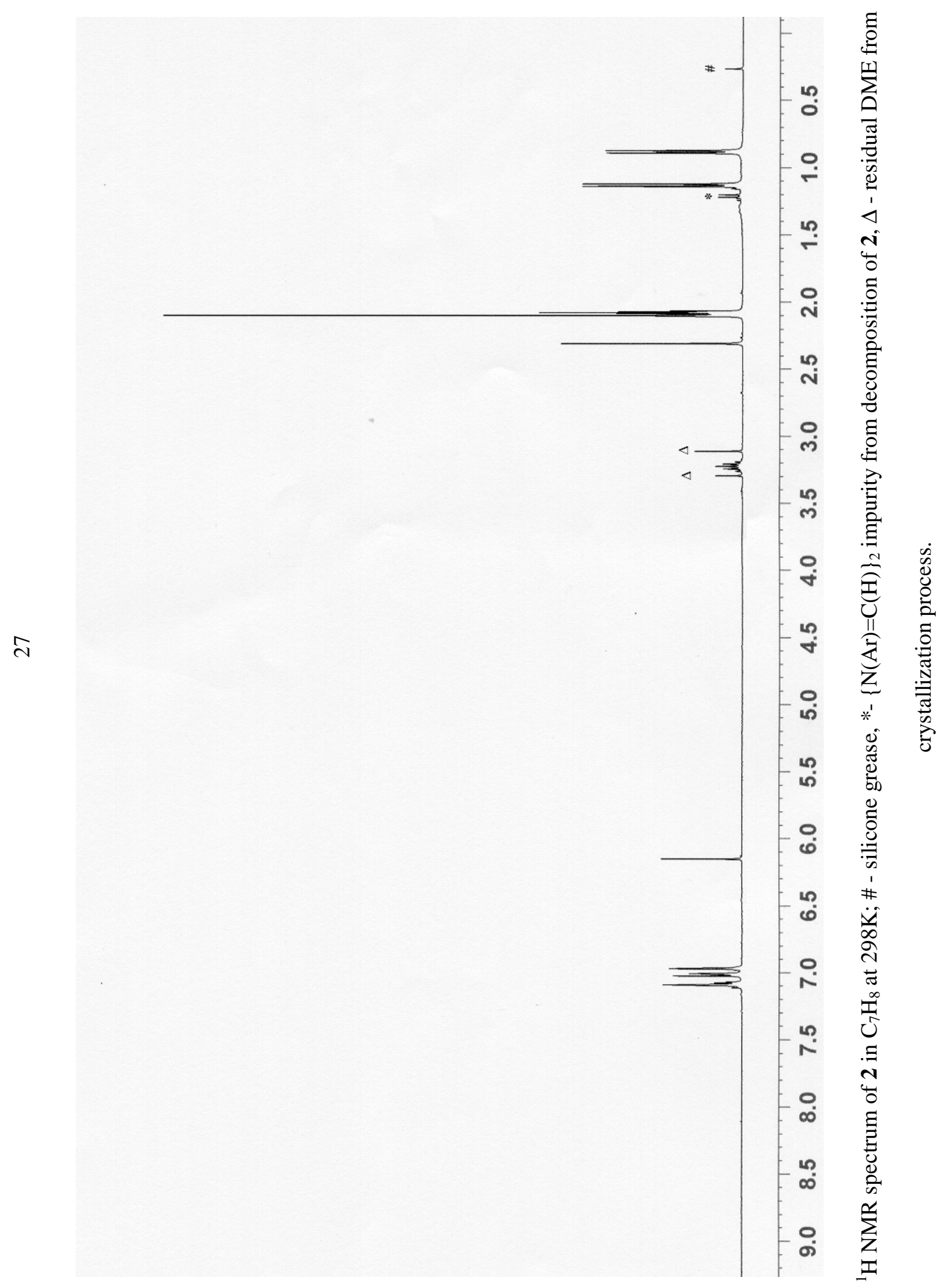

